\title{
Proceedings
}

\section{Communications to the International Sarcopenia Translational Research Conference 2021}

\author{
10 - 11 June 2021 (Virtual)
}

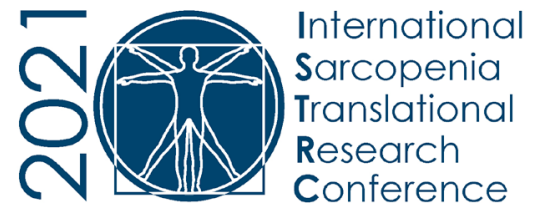

Organising committee for ISTRC 2021

Professor Avan Aihie Sayer (Conference Chair), Matthew Birkbeck, Gayle Cain, Richard Dodds, Antoneta Granic, Leena Habiballa, Christopher Hurst, Eleanor Lockhart, Sian Robinson, Karen Suetterlin, Miles Witham

\section{CLINICAL RESEARCH ABSTRACTS}

\section{STUDY IN NOVEL NEURO-MUSCULAR IMAGING BIOMARKERS FOR MOTOR OUTCOME IN STROKE (SINONIMS)}

Hannah A. Lumley ${ }^{1}$, Mathew Elameer ${ }^{1,3}$, Christopher I. Price ${ }^{1,4}$, Sarah A. Moore ${ }^{4,5}$, Akif Gani ${ }^{3}$, Fiona Smith ${ }^{2}$, Philip English ${ }^{3}$, Helen Rodgers ${ }^{1,4}$, Andrew Blamire ${ }^{2}$, Dipayan Mitra ${ }^{1,3}$

\section{'Population Health Sciences Institute, Newcastle University \\ ${ }^{2}$ Translational and Clinical Research Institute, Newcastle University \\ ${ }^{3}$ Newcastle upon Tyne Hospitals NHS Foundation Trust \\ ${ }^{4}$ Northumbria Healthcare NHS Foundation Trust \\ ${ }^{5}$ Northumbria University}

Introduction: Lower-limb impairment is common after stroke, limiting independence of those affected. Early, accurate prediction of recovery could enable individualised rehabilitation and optimise outcomes. Previous evidence supports an effect of stroke-related damage to corticospinal tracts (CST) on functional recovery. However, effects of post-stroke sarcopenia, resultant muscle fiber-type shift and inflammation are unknown. An algorithm accounting for these changes, based on combined MRI imaging of the source-organ (brain) and end-organ (lower-limb skeletal muscle), may have superior accuracy compared with clinical assessments or isolated MRI biomarkers. We aim to assess the feasibility and utility of MRI biomarkers for predicting lower-limb recovery following stroke.

Methods: 30 patients will be recruited from two North-East England NHS trusts within four weeks of their first-ever ischaemic stroke causing unilateral lower-limb weakness.
Patients undergo brain and thigh muscle MRI, including volumetric sequences, lipid and oedema-based muscle imaging and diffusion tensor imaging of the CST. This is followed by baseline and 3-month clinical assessments of: lower-limb impairment (Fugl-Meyer; Motor NIHSS) strength (handheld-dynamometry) and function (Functional Ambulatory Category).

Results: $10 / 30$ participants have been recruited since 2019 and the study will close in 2021 . Correlations between MRI, clinical and 3-month follow-up outcome data will assess relationships between biomarkers and outcomes to inform a predictive model incorporating baseline clinical and MRI data. Conclusion: This project will provide novel data on sarcopenia-based measures in stroke. If our model has predictive value, a sample size estimate will be calculated to inform future multicentre studies with the aim of developing a robust predictive algorithm.

\section{DIET AND EXERCISE FOR FRAILty (DEFRAIL): THE EFFECT OF A NOVEL COMMUNITY-BASED EXERCISE AND NUTRITIONAL INTERVENTION ON FRAILTY AND RELATED MEASURES IN FRAIL OLDER ADULTS}

Pádraig Bambrick ${ }^{1,2}$, Thomas Byrne ${ }^{3}$, Niamh Phelan', Emma Grant ${ }^{1}$, Ríona Mulcahy ${ }^{1,2}$, Michael Harrison ${ }^{3}$, John Cooke ${ }^{1,2}$

'Department of Geriatric Medicine, University Hospital Waterford, Dunmore Road, Waterford, Republic of Ireland

${ }^{2}$ Royal College of Surgeons in Ireland, 123 St. Stephen's Green, Dublin, Republic of Ireland

${ }^{3}$ Department of Sport \& Exercise Science, Waterford Institute of Technology, Waterford, Republic of Ireland

Correspondence: ISTRC 2021, AGE Research Group, Biomedical Research Building, Campus for Ageing and Vitality, Newcastle-upon-Tyne, UK

E-mail: sarcconf@newcastle.ac.uk 
Background: Frailty in older adults is a growing challenge. There is now an established evidence base for the benefit of exercise and protein supplementation in counteracting frailty, but this has not yet been adequately translated into practice. The aim of this study, Diet \& Exercise for FRAlLty (DEFRAIL), was to develop an effective intervention suitable for widespread uptake. Our first objective was to design an intervention suitable for a wide spectrum of frailty in older adults and deliverable in a community setting. The second was to evaluate its effect on frailty and related measures.

Methods: A modified Delphi process was conducted to develop a draft program, followed by a pilot trial with pre-frail participants. The final intervention (an eight-week program of multicomponent exercise and protein-supplemented milk) was evaluated in a single-group pre-test/post-test study. The primary outcome measure was the Fried frailty criteria (FFC), along with a range of secondary outcome measures. Comparison of change scores over the control period (regular activity) and intervention period (DEFRAIL program) allowed estimation of the treatment effect.

Results: 22 participants ( 16 females, 6 males) completed the intervention (mean age 84.5 years, mean FFC 3.55 prior to the intervention). An improvement of -2.05 points was observed in mean FFC, along with improvements of -3.19 seconds in mean Timed Up \& Go and -1.41 points in mean Geriatric Depression Scale (Short Form) score. No significant change was observed for measures of body mass composition, arterial stiffness, cognition, pain or quality-oflife. Dietary analysis demonstrated increased protein and Vitamin D intake but not carbohydrate or energy intake.

Conclusions: Our preliminary results suggest that the DEFRAIL program is feasible to deliver in an integrated setting and effective at reducing frailty. Given the growing prevalence of frailty, we feel there is an urgent need to incorporate an intervention such as this into public health policy.

\section{EFFECTS OF NICOTINAMIDE ADENINE DINUCLEOTIDE PRECURSORS ON PHYSICAL FUNCTION AND FRAILTY: \\ A SYSTEMATIC REVIEW}

Fred Barker, Ashley Hart, Avan A Sayer, Miles Witham

AGE Research Group. NIHR Newcastle Biomedical Research Centre, Newcastle University and Newcastle Upon Tyne Hospitals NHS Foundation Trust, Newcastle, UK.

Background: Nicotinamide adenine dinucleotide (NAD+) is a key molecule in muscle metabolism and energetics; levels are low in older people with sarcopenia. This systematic review evaluated the effects of NAD+ precursor supplementation on domains of the Fried frailty phenotype in humans.

Methods: We included randomised controlled trials assessing outcomes relevant to the Fried frailty phenotype domains: slowness; weakness; exhaustion; low physical activity and weight loss. All stages of the review were conducted independently by two separate authors. A systematic search strategy was used on several databases (MEDLINE, EMBASE, CINAHL, CENTRAL, ISRCTN, clinicaltrials.gov, NHS e-Library and Google Scholar) to find appropriate studies. Risk of bias was assessed using the Cochrane Risk-of-Bias 2 tool. Results were grouped by phenotypic domain and described through narrative synthesis.

Results: Twenty studies met the inclusion criteria; size ranged from 2 to 77 participants. No studies assessed frailty as an outcome in itself. Studies used a range of interventions; nicotinamide riboside $(n=5)$ and acipimox $(n=5)$ were the most commonly used. Most studies examined short-term interventions up to 6 months prior to outcome measurement, with 10/20 interventions lasting 1 week or less. A wide range of outcomes were measured with considerable heterogeneity precluding meta-analysis. Study populations were also heterogenous; only 3 studies enrolled participants with a mean age $>65$ years. Risk of bias analysis found an unclear or high risk of bias in all but one study. There was no clear pattern as to whether NAD+ precursors were better than control for any domain of the frailty phenotype; the majority of studies reported null findings for most outcomes. Conclusion: The current literature on NAD+ precursor supplementation is insufficient to ascertain effects on physical performance and the frailty phenotype. High-quality studies are required to explore the effect of NAD+ precursor supplementation, particularly in older people who are most likely to benefit.

\section{CONNECTING DIGITAL MOBILITY ASSESSMENT TO SARCOPENIA AND FRAILTY: THE MOBILISE-D STUDY}

Alison J. Yarnall ${ }^{1,2}$, Claudia Mazzà ${ }^{3}$, Arne Mueller ${ }^{4}$, Brian Caulfield ${ }^{5}$, Marie McCarthy ${ }^{6}$, Clemens Becker ${ }^{7}$, Ram Miller ${ }^{4}$, Marco Viceconti, ${ }^{8,9}$, Wim Dartee ${ }^{4}$, Judith Garcia-Aymerich ${ }^{10}$, Hans Guehring ${ }^{11}$, Beatrix Vereijken ${ }^{12}$, Valdo Arnera ${ }^{13}$, Nadir Ammour ${ }^{14}$, Tilo Hache ${ }^{4}$, Mike Jackson ${ }^{15}$, Ronenn Roubenoff ${ }^{4}$, Lynn Rochester ${ }^{1,2}$

'Translational and Clinical Research Institute, Faculty of Medical Sciences, Newcastle University, Newcastle upon Tyne, UK

${ }^{2}$ NIHR Newcastle Biomedical Research Centre, Newcastle upon Tyne Hospitals NHS Foundation Trust and Faculty of Medical Sciences, Newcastle University, Newcastle upon Tyne, UK ${ }^{3}$ INSIGNEO Institute for in silico Medicine, University of Sheffield, Sheffield, UK

${ }^{4}$ Novartis Institutes for BioMedical Research, Basel, Switzerland

5 Insight Centre for Data Analytics, University College Dublin, Ireland ${ }^{6}$ CON Clinical Research Limited, Ireland

${ }^{7}$ Robert Bosch Hospital, Stuttgart, Germany

${ }^{8}$ Department of Industrial Engineering, Alma Mater Studiorum University of Bologna, Italy

${ }^{9}$ Medical Technology Lab, IRCCS Istituto Ortopedico Rizzoli, Bologna, Italy 
10/SGlobal, Barcelona, Spain

"Merck KgaA, Frankfurter, Germany

${ }^{12}$ Department of Neuromedicine and Movement Science, Faculty of Medicine and Health Sciences, Norwegian University of Science and Technology (NTNU), Trondheim, Norway

${ }^{13}$ Eresearch Technology Inc, USA

${ }^{14}$ Sanofi Research and Development, Chilly-Mazarin, France

${ }^{15}$ xscient Limited, UK

Background: The increasing longevity of the world's population together with prolonged survival in many chronic diseases means that more people are suffering from loss of mobility. Efforts to mitigate this loss of mobility are an increasing priority and promising interventions are now under investigation. To target mobility loss effectively and thus be able to prevent it, we need valid tools that can detect and measure it in daily life. It is also of vital importance that mobility outcomes are assessed in relation to other ageing syndromes, including sarcopenia and frailty. Digital technology has the potential for measuring and monitoring digital mobility outcomes in the real world, but as yet there are no robust, validated technology solutions.

Methods: The overarching objectives of Mobilise-D are to: 1) deliver a valid solution for real-world digital mobility assessment; 2) validate digital mobility outcomes in predicting clinical outcome in a variety of disease states including Parkinson's disease, chronic obstructive pulmonary disease, multiple sclerosis, and proximal femoral fracture; and 3) obtain regulatory and health stakeholder approval for digital mobility assessment.

Results: "Connecting digital mobility assessment to clinical outcomes for regulatory and clinical endorsement" (Mobilise-D), funded by the European Innovative Medicines Initiative 2 Joint Undertaking, includes 34 international research partners based at leading international universities and some of the world's largest pharmaceutical and technical companies. Mobilise-D started on 1 April 2019 and will run for a duration of five years. A number of measures of mobility and disability will be collected, and a sub-study on sarcopenia and frailty will be conducted within each disease cohort.

Conclusions: Mobilise-D's results will directly facilitate drug development and establish the roadmap for clinical implementation of new, complementary tools to identify, stratify and monitor disability, thereby enabling widespread, cost-effective access to optimal clinical mobility management through personalised healthcare.

\section{SARCOPENIC OBESITY IN NEURODISABILITIES: THE SarcObeNDS STUDY}

Yannis Dionyssiotis', Maria-Christina Papadatou', Antonios Galanos ${ }^{2}$, Eleni Lazarou ${ }^{3}$, Prodromos Kosmidis ${ }^{3}$, Evangelia Christaki ${ }^{3}$, Christina Papanastasiou ${ }^{1}$, Nikolaos Groumas'

$11^{\text {st }}$ Physical Medicine and Rehabilitation Department, National Rehabilitation Center EKA, Athens, Greece
'Laboratory for Research of the Musculoskeletal System, National and Kapodistrian University of Athens, Kifissia, Greece

${ }^{3}$ Radiology Department, National Rehabilitation Center EKA, Athens, Greece

Background: The investigation of sarcopenic obesity (SO) and sarcopenia is important in populations with disabilities as they lead to metabolic disorders and concomitant diseases that aggravate the neurological status. The study was designed to investigate $\mathrm{SO}$ and sarcopenia in people with neurological disabilities (stroke, spinal cord injury, traumatic brain injuries etc.), to describe the frequency and thresholds for SO in neurodisabled persons and the fat and lean mass distribution compared to able-bodied persons.

Methods: This is the SarcObeNDS observational study including hospitalized patients and controls ( 100 and 30 , respectively), aged $60 \pm 14.22$, measured with whole body DXA (Lunar). SO was assessed with Total body Fat (\%) (TBF), and Fat mass/height ${ }^{2}\left(\mathrm{FM} / \mathrm{h}^{2}, \mathrm{Kg} / \mathrm{m}^{2}\right)$ and sarcopenia with Appendicular muscle mass $/ \mathrm{height}^{2}\left(\mathrm{Kg} / \mathrm{m}^{2}\right)$ - skeletal muscle index (SMI). The study has been registered in ClinicalTrials.gov with unique number: NCT03863379.

Results: We found statistically significant results for body mass index (BMI) $(p=0.012)$ and SMI (7.18 \pm 0.95 vs. $6.00 \pm 1.13$, $\mathrm{p}<0.001$ ) between able-bodied and neurodisabled people. No statistical significance was found in TBF $(p=0.793)$ and FM/ $h^{2}(p=0.143)$. No significant interaction between the factors "group" and "sex" for the index TBF and SMI was found $(p=0.832$ and $p=0.889$, respectively). A strong positive significant correlation was found between BMI and TBF and FM in able-bodied and neurodisabled people $(r=0.616, p<0.001$ vs. $r=0.728, p<0.001$ and $r=0.420, p<0.001$ vs. $r=0.868$, $\mathrm{p}<0.001$, respectively).

Conclusions: There are differences in able-bodied and neurodisabled people regarding sarcopenia and SO indices. Values below current SMI threshold for sarcopenia indicate that sarcopenia is a real complication in these populations. At the clinical level, the recognition of these diseases will prevent metabolic disorders in these frail populations.

\section{EFFECT OF PERINDOPRIL ON PHYSICAL PERFORMANCE, MUSCLE MASS AND QUALITY OF LIFE IN OLDER PEOPLE WITH SARCOPENIA: RESULTS FROM THE LACE RANDOMISED CONTROLLED TRIAL}

Miles D. Witham ${ }^{1,2}$, Simon Adamson ${ }^{3}$, Alison Avenell4, Margaret M. Band ${ }^{3}$, Peter T. Donnan ${ }^{3}$, Jacob George ${ }^{2}$, Adrian Hapca ${ }^{3}$, Cheryl Hume ${ }^{3}$, Paul Kemp ${ }^{5}$, Emma McKenzie ${ }^{3}$, Kristina Pilvinyte ${ }^{3}$, Karen Smith ${ }^{3}$, Allan D. Struthers ${ }^{2}$, Deepa Sumukadas ${ }^{6}$ for the LACE study group

'AGE Research Group, NIHR Newcastle Biomedical Research Centre, Newcastle University and Newcastle-upon-Tyne Hospitals Trust, Newcastle-upon-Tyne, UK 
${ }^{2}$ Molecular and Clinical Medicine, University of Dundee, Dundee, UK ${ }^{3}$ Tayside Clinical Trials Unit, University of Dundee, Dundee, UK ${ }^{4}$ Health Services Research Unit, University of Aberdeen, Aberdeen, UK ${ }^{5}$ Cardiovascular and Respiratory Interface Section, National Heart and Lung Institute, Imperial College London, London, UK.

${ }^{6}$ Department of Medicine for the Elderly, NHS Tayside, Dundee, UK

Background: Angiotensin-converting enzyme inhibitors such as perindopril have been proposed as treatments to improve muscle mass and physical performance but have not been tested in randomised controlled trials enrolling patients with sarcopenia.

Methods: We conducted a placebo-controlled, parallel group, double-blind, randomised two-by-two factorial trial. Participants aged 70 and over with low muscle mass by bioimpedance and either low grip strength or low walk speed, were recruited from $14 \mathrm{UK}$ sites. Participants were randomised to receive one year of perindopril $4 \mathrm{mg}$ once daily or matching placebo, and to receive leucine powder $2.5 \mathrm{~g}$ three times a day or matching placebo. The primary outcome was the betweengroup difference in the Short Physical Performance Battery, measured at baseline, 6 and 12 months, analysed using repeated-measures mixed models. Secondary outcomes included grip strength, quadriceps strength, six-minute walk distance, appendicular muscle mass measured by dual $\mathrm{x}$-ray absorptiometry, quality of life measured using the EQ5D tool, falls rates and adverse events.

Results: We screened 320 people and randomised 145 participants, mean age 79 (SD 6) years; 78 (54\%) were women and the mean SPPB was 7.0 (SD 2.4). 73 were randomised to perindopril and 72 to placebo. Median adherence was lower for perindopril ( $76 \%$ vs $96 \%$; $p<0.001$ ). Perindopril had no significant effect on the primary outcome (adjusted treatment effect -0.1 points [95\% $\mathrm{Cl}-1.2$ to 1.0$]$ ). No significant treatment effect was seen for any secondary outcome except for worse EQ5D thermometer scores in the perindopril group (treatment effect -12 points [95\% $\mathrm{Cl}-21$ to -3$]$ ). More adverse events were seen in the perindopril group ( 218 vs 165 ) but falls rates were similar (perindopril $2.0[95 \% \mathrm{Cl} 1.1$ to 3.0$]$ per year; placebo 2.8 [ $95 \% \mathrm{Cl} 0.6$ to 5.1 ] per year).

Conclusion: Perindopril did not improve measures of physical performance, muscle mass or quality of life in older people with sarcopenia.

\section{EFFECT OF LEUCINE SUPPLEMENTATION ON PHYSICAL PERFORMANCE, MUSCLE MASS AND QUALITY OF LIFE IN OLDER PEOPLE WITH SARCOPENIA: RESULTS FROM THE LACE RANDOMISED CONTROLLED TRIAL}

Miles D. Witham ${ }^{1,2}$, Simon Adamson ${ }^{3}$, Alison Avenell, Margaret M. Band ${ }^{3}$, Peter T. Donnan ${ }^{3}$, Jacob George ${ }^{2}$, Adrian Hapca ${ }^{3}$, Cheryl Hume ${ }^{3}$, Paul Kemp ${ }^{5}$, Emma McKenzie $^{3}$, Kristina Pilvinyte ${ }^{3}$, Karen Smith ${ }^{3}$, Allan D. Struthers ${ }^{2}$, Deepa Sumukadas ${ }^{6}$ for the LACE study group
'AGE Research Group, NIHR Newcastle Biomedical Research Centre, Newcastle University and Newcastle-upon-Tyne Hospitals Trust, Newcastle-upon-Tyne, UK

${ }^{2}$ Molecular and Clinical Medicine, University of Dundee, Dundee, UK ${ }^{3}$ Tayside Clinical Trials Unit, University of Dundee, Dundee, UK

${ }^{4}$ Health Services Research Unit, University of Aberdeen, Aberdeen, UK ${ }^{5}$ Cardiovascular and Respiratory Interface Section, National Heart and Lung Institute, Imperial College London, London, UK

${ }^{6}$ Department of Medicine for the Elderly, NHS Tayside, Dundee, UK

Background: Leucine supplementation improves muscle protein synthesis in physiological studies and has been proposed as a treatment to improve muscle mass and physical performance. We tested the effects of leucine supplementation in a randomised controlled trial enrolling patients with sarcopenia.

Methods: We conducted a placebo-controlled, parallel group, double-blind, randomised two-by-two factorial trial. Participants aged 70 and over with low muscle mass by bioimpedance and either low grip strength or low walk speed, were recruited from 14 UK sites. Participants were randomised to receive one year of leucine powder $2.5 \mathrm{~g}$ three times a day or matching placebo, plus perindopril $4 \mathrm{mg}$ once daily or matching placebo. The primary outcome was the between-group difference in the Short Physical Performance Battery, measured at baseline, 6 and 12 months, analysed using repeated-measures mixed models. Secondary outcomes included grip strength, quadriceps strength, sixminute walk distance, appendicular muscle mass measured by dual $\mathrm{x}$-ray absorptiometry, quality of life measured using the EQ5D tool, falls rates and adverse events.

Results: We screened 320 people and randomised 145 participants, mean age 79 (SD 6) years; 78 (54\%) were women and the mean SPPB was 7.0 (SD 2.4). 72 were randomised to leucine and 73 to placebo. Median adherence was the same in both groups ( $76 \%$ vs $76 \% ; p=0.99$ ). Leucine had no significant effect on the primary outcome (adjusted treatment effect 0.1 points [ $95 \% \mathrm{Cl}-1.0$ to 1.1$]$ ]). No significant treatment effect was seen for any secondary outcome. There were similar numbers of adverse events in both groups (leucine 187, placebo 196) and falls rates were similar (leucine $1.9[95 \% \mathrm{Cl} 0.9$ to 2.9$]$ per year; placebo $2.9[95 \% \mathrm{Cl} 0.8$ to 5.0$]$ per year).

Conclusion: Leucine did not improve measures of physical performance, muscle mass or quality of life in older people with sarcopenia.

\section{POOR APPETITE IS ASSOCIATED WITH LOWER MUSCLE STRENGTH IN COMMUNITY DWELLING OLDER ADULTS}

Natalie J. Cox ${ }^{1,2}$, Avan A. Sayer ${ }^{3,4}$, Helen C. Roberts ${ }^{1,2,5}$, Sian M. Robinson ${ }^{3,4}$

'Academic Geriatric Medicine, Faculty of Medicine, University of Southampton, Tremona Road, Southampton, UK 
${ }^{2}$ NIHR Southampton Biomedical Research Centre, University of Southampton and University Hospital Southampton NHS Foundation Trust, Southampton, UK

${ }^{3}$ AGE Research Group, Translational and Clinical Research Institute, Newcastle University, Newcastle upon Tyne, UK

${ }^{4}$ NIHR Newcastle Biomedical Research Centre, Newcastle upon Tyne Hospitals NHS Foundation Trust and Newcastle University, Newcastle upon Tyne, UK

${ }^{5}$ NIHR Applied Research Collaborative (ARC) Wessex, University of Southampton, Southampton, UK

Background: Anorexia of ageing is common but often not assessed in clinical practice despite the availability of simple tools such as the four-item Simplified Nutritional Appetite Questionnaire (SNAQ). Anorexia of ageing is associated with altered dietary pattern and weight loss, potentially contributing to the development of sarcopenia through loss of muscle mass. However, the association between appetite and muscle strength is less clear.

Methods: A cross sectional observational study of community dwelling adults aged over 60 years, attending outpatient appointments in one hospital. Appetite was assessed using SNAQ; muscle strength by handgrip strength with a Jamar dynamometer and standard protocol. The association between appetite (SNAQ score between 5 and 20) and grip strength was assessed in unadjusted and adjusted continuous analyses.

Results: The study included 86 participants, $62 \%$ female with mean age 77.5 (8.1) years (range 60-93), median of four comorbidities (IQR 3-6), grip strength 30.0 (SD 8.7) $\mathrm{kg}$ for males, 19.3 (SD 6.6) kg for females. The mean SNAQ score was 15.0 (SD 8.1). SNAQ score was associated with grip strength: poorer appetite was associated with lower grip strength (unadjusted coefficient 1.59; $p=0.003$ ). This association remained when adjusted for sex (adjusted coefficient $0.433 ; p=0.013$ ). The association was not robust to adjustment for age and comorbidity count.

Conclusions: We observed an association between poorer appetite and lower muscle strength in community dwelling older adults, independent of sex. This association was not robust when accounting for effects of age and comorbidity. Poor appetite may be associated with low muscle strength prior to older age and comorbidities but further investigation is required. Appetite is simple to assess in older people using SNAQ and may prove useful in earlier identification of individuals at risk of developing sarcopenia.

\section{ASSESSING CHANGES IN PHYSICAL ACTIVITY IN COMMUNITY DWELLING OLDER ADULTS BEFORE AND AFTER COVID-19 RESTRICTIONS IN THE UNITED KINGDOM}

Matthew G. Birkbeck ${ }^{1,2,3}$, Susan Hillman', Christopher Hurst ${ }^{1}$, Antoneta Granic ${ }^{1}$, Terry Aspray', Avan A. Sayer ${ }^{1,2}$, Richard M. Dodds ${ }^{1}$
'Newcastle University Translational and Clinical Research Institute (NUTCRI), Newcastle University, Newcastle upon Tyne

${ }^{2}$ Newcastle NIHR Biomedical Research Centre, Newcastle University, Newcastle upon Tyne

${ }^{3}$ Northern Medical Physics \& Clinical Engineering, Newcastle upon Tyne NHS FT, Newcastle upon Tyne

Introduction: Higher levels of physical activity (PA) may be important in the prevention of sarcopenia. The coronavirus pandemic (COVID-19) caused governments to introduce measures including self-isolation, social distancing and restrictions on movement within periods of national and local lockdowns. It is therefore of interest to study the impact of restrictions on PA behaviour in older people. Most studies to date have focussed on subjective assessment of PA. We performed a preliminary objective assessment of changes in PA associated with these restrictions in a group of participants from Muscle Ageing and Sarcopenia Study (MASS_Lifecourse).

Methods: PA was measured in 9 volunteers (mean age: 59) on two occasions; before restrictions $(08 / 11 / 2018-$ $02 / 03 / 2020)$ and during restrictions $(10 / 11 / 2020-$ $06 / 03 / 2021$ ). Participants wore a wrist-worn tri-axial accelerometer for 7 days. Minutes per day spent in moderatevigorous PA (MVPA) and mean acceleration per hour ( $\mathrm{mg} / \mathrm{h}$ ), were derived from recordings. The mean ratio of MVPA pre/post restrictions was calculated to assess differences between occasions. Mean acceleration (MA) at each occasion was compared using correlation analysis.

Results: The 9 volunteers consisted of 3 females and 6 males. The median time between assessments was 16 months (min: $11 \mathrm{~m}$, max: $24 \mathrm{~m}$ ). Change in MVPA expressed as the mean of the ratio of MVPA pre/post restrictions 0.98 . The correlation between MA pre/post restrictions was strong $\left(R^{2}=0.92 ; p<0.0001\right)$.

Conclusions: Mean ratio of MVPA between pre/post restrictions is close to 1, suggesting no substantial change in MVPA in this population due to restrictions. Post restrictions, we saw a lower peak in MA around 10 am and evidence of increased activity around the middle of the day $(2 \mathrm{pm})$. However, patterns of PA were significantly correlated suggesting no overall change. Data across a wider age range are being collected as part of an ongoing study to fully assess the impact of restrictions on PA.

\section{QUANTIFYING THE INTERNAL TRAINING RESPONSE TO RESISTANCE EXERCISE IN OLDER ADULTS: FINDINGS FROM THE MILK INTERVENTION MUSCLE AGEING (MIIKMAN) PILOT STUDY}

Christopher Hurst ${ }^{1,2}$, Lorelle Dismore ${ }^{1,2,3}$, Avan A. Sayer ${ }^{1,2}$, Emma Stevenson ${ }^{4,5}$, Terry Aspray ${ }^{1,2,6}$, Antoneta Granic ${ }^{1,2}$ 'AGE Research Group, Translational and Clinical Research Institute, Faculty of Medical Sciences, Newcastle University, UK ${ }^{2}$ NIHR Newcastle Biomedical Research Centre, Newcastle upon Tyne Hospitals NHS Foundation Trust and Newcastle University, UK 
${ }^{3}$ Northumbria Healthcare NHS Foundation Trust, North Tyneside Hospital, North Shields, UK

${ }^{4}$ Population Health Sciences Institute, Faculty of Medical Sciences, Newcastle University, UK

${ }^{5}$ Human Nutrition Research Centre, Newcastle University, UK

${ }^{6}$ Newcastle upon Tyne Hospitals NHS Foundation Trust, Musculoskeletal Unit, Freeman Hospital, Newcastle upon Tyne, UK

Background: Resistance exercise (RE) is an appealing strategy for counteracting the deleterious consequences of sarcopenia including reduced muscle strength and physical performance. However, as exercise induced adaptation is primarily driven by the internal training response (i.e., the physiological stress imposed by the training dose), eliciting a consistent and standardised training dose is necessary to fully evaluate potential intervention effects.

Methods: Thirty community-dwelling older adults ( $71.7 \pm 3.6$ years; 12 female) naïve to $\mathrm{RE}$, performed 6 weeks of twice weekly, machine-based progressive RE involving a combination of upper- and lower-body exercises (2-3 sets of $8-10$ repetitions at $70-80 \% 1$ repetition maximum). Internal training response was quantified using ratings of perceived exertion (RPE). Following each RE session, participants provided an overall rating of perceived exertion (SRPE) and differential ratings of perceived exertion for upper-body muscle exertion (SRPE-U) and lower-body muscle exertion (SRPE-L) using the CR 100 scale (range 0-100 arbitrary units [AU]; 12, easy; 22, moderate; 35 , somewhat hard; 50 , hard; 100 , maximal). Data were analysed using mixed linear models.

Results: Twenty-nine participants completed the intervention with an overall attendance of $97 \%$ (338 out of a possible 348 sessions attended). Mean RE session intensity was rated as between 'somewhat hard' and 'hard' for all RPE types (SRPE: $38 \pm 19$, sRPE-U: $41 \pm 21$, sRPE-L: $35 \pm 19$ ). There was no change in mean RPE throughout the intervention (sRPE, mean slope: $-2 ; 95 \%$ confidence interval [CI] -9 to 4 , sRPE-U: $-2[-9,5]$, SRPE-L: $-3[-10,3])$, despite substantial variability between participants (SRPE, slope SD: 15; 95\% Cl [10, 21], SRPE-U 14 [9, 21], SRPE-L 15 [11, 21]).

Conclusions: RE induced a substantial internal training response with mean ratings of perceived exertion remaining consistent throughout the intervention. These metrics should be validated against objective measures of $\mathrm{RE}$ response such as improved muscle function.

\section{THE EFFECTS OF RESISTANCE EXERCISE TRAINING ON MITOCHONDRIAL DYSFUNCTION IN MITOCHONDRIAL MYOPATHY PATIENTS}

Valeria Di Leo, Jane Newman, Fiona Robertson, Conor Lawless, Sarah Pickett, Gavin Hudson, Tiago Gomes, Grainne S. Gorman, Helen A. Tuppen, Amy E Vincent, Oliver M. Russell

Wellcome Centre for Mitochondrial Research, Translational and Clinical Research Institute, Newcastle University, UK
Background: Patients with single, large-scale mitochondrial DNA (mtDNA) deletions often present with mitochondrial myopathy for which no treatment is available. Exercise intervention induces beneficial effects in mitochondrial myopathy; however, we do not yet understand the mechanism for this.

Methods: This study investigates biochemical, genetic and molecular changes triggered by resistance exercise training to highlight potential exercise modulator(s) for mitochondrial myopathy patients. Pre- and post-exercise skeletal muscle biopsies were taken from a cohort of single, large-scale mtDNA deletion patients after 16 weeks of supervised highintensity resistance exercise training. Biopsies were assessed for changes in oxidative phosphorylation, heteroplasmy, fibre type and size and differentially expressed (DE) genes.

Results: All patients improved aerobic capability after exercise, especially P1 and P5 who also showed a significant increase of the VDAC1 mean distribution $(p<0.0001)$, therefore suggesting a positive correlation between mitochondrial mass and aerobic capability $\left(R^{2}=0.42\right.$, $p=0.04$ ). Furthermore, all post-exercise biopsies showed an increase of fibres with higher MTCO 1 level (P1, P2 and P3 $\mathrm{p}<0.0001 ; \mathrm{P} 4 \mathrm{p}=0.0015$; P5 $\mathrm{p}=0.0012$ ). Heteroplasmy decreased in three patients after exercise $(P 1 p=0.001)$. Interestingly, fibre type distribution switched towards oxidative fibre types Myh7+ and Myh1+ in the majority of patients ( $P 1$ and $P 4 p=0.01, P 2 p<0.0001$ ). In addition, all patients except $P 2$ showed significant hypertrophy in fibre type Myh7+ $(p<0.0001)$, whereas P2, P3 and P4 showed hypertrophy in fibre type Myh1+ $(p=0.0007)$. Finally, through RNA-sequencing analysis we identified a list of $D E$ genes between pre- and post-exercise cases.

Conclusion: Resistance exercise training is effective in mitochondrial myopathy patients inducing rescue of complex IV deficiency and metabolic oxidative shift in skeletal muscle. Transcriptomic changes were triggered after exercise, which will be confirmed through Imaging Mass Cytometry.

\section{LOWER PSOAS MUSCLE AREA IS ASSOCIATED WITH INCREASED MORTALITY AFTER ENDOVASCULAR ANEURYSM REPAIR IN OLDER ADULTS}

\author{
J. Ting', E. Zhang', K. Chan', J. Lu', W. Raymond', \\ R. Kwok', S. Jansen ${ }^{1,2}$, C. Inderjeeth ${ }^{1}$ \\ 'Sir Charles Gairdner Hospital, Perth, Australia \\ ${ }^{2}$ Heart and Vascular Research Institute, Perth, Australia
}

Background: Psoas muscle area (PMA) is an easily measured surrogate marker of sarcopenia. However, the evidence is conflicted as to whether PMA is associated with poorer postoperative survival, post-operative outcomes, readmissions, discharge destination or increased length of stay. This study aimed to re-explore the relationship between PMA and outcomes post asymptomatic infrarenal endovascular aortic 
repair of aneurysm (EVAR) in older adults.

Methods: A retrospective observational study reviewed people aged over 65 years undergoing EVAR for asymptomatic infrarenal AAA from $1 / 1 / 2013$ to $31 / 12 / 2017$. Patients with connective tissue disease, mycotic or inflammatory, emergency or non-elective cases including symptomatic AAA were excluded. PMA was measured at mid L4 vertebral body on preoperative computer tomography scan. Primary outcome was survival in days from operation. Secondary outcomes were 30-day readmissions related to EVAR, post-operative complications and length of stay measured in days.

Results: 97 patients (mean age 77.5 years, $78 \%$ male) were assessed. After adjusting for age, sex and BMI, patients in the lowest PMA tertile had an increased 5-year mortality (HR 2.76, 95\% $\mathrm{Cl} 1.08,7.03 ; \mathrm{p}=0.034$ ). After adjusting for BMI patients with the lowest tertiles of PMA for either sex had increased total LOS $(\beta \quad 0.33,95 \% \mathrm{Cl} 0.04,0.63$; $\mathrm{p}=0.028$ ) and odds of dying within 5 -years (OR 3.53, $95 \% \mathrm{Cl} 1.21,10.27: \mathrm{p}=0.021$ ). No significant associations were found between the lowest tertile of PMA of either sex and readmissions, discharge destination and complications. Conclusion: The lowest tertile of PMA, a surrogate marker for sarcopenia, was associated with increased mortality in patients undergoing EVAR for asymptomatic infrarenal AAA. An association with lowest tertile of PMA and slightly increased length of stay was found however this was of uncertain clinical significance. Although sarcopenia is a complex phenomenon and the literature surrounding it is still evolving, PMA may form one component of risk assessment in patients undergoing EVAR.

\section{AGE-RELATED CHARACTERISTICS OF SKELETAL MUSCLE HEALTH ACROSS 18-70 YEARS}

Isobel Thornley', Gareth Jones', Peter Francis ${ }^{3}$,

Mark I. Johnson ${ }^{2}$

'Musculoskeletal Research Group, Leeds Beckett University, Leeds, United Kingdom

${ }^{2}$ Centre for Pain Research, Leeds Beckett University, Leeds, United Kingdom

${ }^{3}$ Department of Science and Health, Institute of Technology Carlow, Carlow, Ireland

Background: There is a paucity of cross-sectional research on muscle morphology, function, and functional capability in all age-bands of healthy adults. Therefore, age-related differences in skeletal muscle across the adult lifespan are unknown. The aim of this study was to evaluate indices of muscle morphology, function and functional capability in a sample of healthy adults aged 18-70 yrs.

Methods: We conducted a cross-sectional study of 274 healthy participants between 18 and 70 years that used $B$ mode Ultrasonography and Tensiomyography to measure muscle thickness, pennation angle, fascicle length, and contractile properties in Vastus Lateralis. We also measured grip strength (handheld dynamometry) and functional capability ( 1 -minute chair rise test). Data analysis included descriptive statistics, correlations, one-way ANOVAs, and multiple regressions.

Results: Age was negatively associated with muscle thickness ( $r s=-0.56$ ), pennation angle ( $r s=-0.50$ ), fascicle length ( $r s=$ -.30), maximal displacement of an involuntary contraction $(r=-0.24)$, grip strength ( $r s=-0.27)$ and the 1 -minute chair rise test ( $r s=-0.32$ ). Age was positively associated with echo intensity of the muscle ( $r s=0.40$ ) and total contraction time of an involuntary contraction ( $r s=0.20$ ). ANOVA and post hoc analysis revealed differences in muscle morphology, function and functional capability between the 18-29 age band and the 50-59 and 60-70 age bands. The interaction of age and level of physical activity better predicted changes in the variables $\left(r^{2}=0.04-0.32\right)$, compared to either age $\left(r^{2}=0.04-0.30\right)$ or physical activity alone $\left(r^{2}=0.03-0.10\right)$. Conclusion: There is a decline in muscle thickness across the adult lifespan and the differences may increase at approximately 50 yrs. These changes in muscle thickness may be reflected in concurrent changes in morphology, function, and functional capability. Physical activity likely acts to abate detrimental change and maintain functional capacity for activities of daily living.

\section{IDENTIFYING INCIDENTAL BIOMARKERS OF SARCOPENIA FROM ROUTINE IMAGING IN A MEMORY CLINIC COHORT}

\section{Sophia Avramoudas ${ }^{1}$, Shehani Wickramasinghe ${ }^{1}$, Nan Jordan', Matthew Gvalda', Paul Yates ${ }^{2}$ \\ 'The University of Melbourne, Melbourne, Australia \\ ${ }^{2}$ Continuing Care department, Austin Health, Heidelberg, Australia}

Background: Sarcopenia is shown to be associated with cognitive impairment, and evidence suggests that physical frailty influences the clinical expression of dementia. There is also growing interest in exploring novel biomarkers for sarcopenia. This study sought to assess the feasibility of measuring average temporalis muscle thickness (TMTavg) on routine brain imaging as a potential biomarker of sarcopenia, and to explore the relationship between temporalis muscle measurements and frailty, as assessed using a Frailty Index (FI), in a memory clinic cohort.

Methods: This was a retrospective study in which TMT was analysed on coronal, T1-weighted MR images of the brain sourced from 111 patients assessed at a tertiary memory clinic in 2014. The relationship between TMTavg and FI was investigated using Spearman correlation test, and Mann-Whitney $U$ test to compare the distribution of TMTavg between frail and non-frail patients (frail defined as $\mathrm{FI} \geq 2.5$ ). Results: The median age of patients was $76.4 ; 47$ men and 64 women. 26 (23.4\%) were diagnosed with Mild Cognitive Impairment, 37 (33.3\%) with dementia, 10 (9.0\%) with another diagnosis, and $38(34.2 \%)$ received no formal 
diagnosis. 38 (34.2\%) participants fell into the frail group, and $73(65.8 \%)$ not-frail. Median TMTavg was $5.3 \mathrm{~mm}$ (IQR: $4.3 \mathrm{~mm}-6.6 \mathrm{~mm}$ ). Spearman correlation test demonstrated significant negative correlation between TMTavg and frailty ( $F I)(r h o=-0.24, p=0.01)$, and Mann-Whitney $U$ test revealed significant difference $(Z=-3.341, p=0.001)$ in distribution pattern of TMTavg between not-frail (median=5.7 mm; IQR=4.8 - 6.8) and frail (4.5 mm; 4.0-5.6) groups.

Conclusion: TMT is easily assessed on routine brain imaging, and there is significant association between TMT and frailty as assessed using the Frailty Index. This supports the hypothesis that increased TMT is associated with decreased frailty in this cohort and may act as a radiological biomarker of sarcopenia.

\section{THE MOVESTRONG PROGRAM FOR PROMOTING BALANCE AND FUNCTIONAL STRENGTH TRAINING AND ADEQUATE PROTEIN INTAKE IN PRE-FRAIL OLDER ADULTS: A PILOT RANDOMIZED CONTROLLED TRIAL}

Isabel B. Rodrigues' ${ }^{1}$, Justin B. Wagler ${ }^{1}$, Heather Keller ${ }^{1,2}$, Lehana Thabane ${ }^{3}$, Maureen C. Ashe ${ }^{4}$, Sheila Brien ${ }^{5}$, Angela Cheung $^{6}$, Larry Funnell ${ }^{5}$, Ravi Jain ${ }^{5}$, Desmond Loong ${ }^{7}$, Wanrudee Isaranuwatchai ${ }^{7,8}$, Jamie Milligan', Marina Mourtzakis', Alexandra Papaioannou ${ }^{9}$, Sharon Straus ${ }^{7,10}$, Zachary J. Weston ${ }^{11,12}$, Lora M. Giangregorio ${ }^{1,2}$

'Department of Kinesiology, University of Waterloo, Waterloo, ON, Canada, N2L 3G1

${ }^{2}$ Schlegel-UW Research Institute for Aging, University of Waterloo, Waterloo, ON, Canada, N2J OE2

${ }^{3}$ Department of Health Research Methods, Evidence and Impact, McMaster University, Hamilton, ON, Canada, L8S 4L8

${ }^{4}$ Department of Family Practice, University of British Columbia, Vancouver, BC, Canada, V6T 1 Z3

${ }^{5}$ Canadian Osteoporosis Patient Network, Osteoporosis Canada, Toronto, ON, Canada, M3C $1 \mathrm{H} 9$

${ }^{6}$ Department of Medicine, University Health Network, University of Toronto, Toronto, ON, Canada, L8S 4L8

${ }^{7}$ CLEAR Health Economics, Knowledge Translation Program, Li Ka Shing Knowledge Institute, St. Michael's Hospital, Unity Health Toronto, Toronto, ON, Canada, M5B 1 T8

8Institute of Health Policy, Management \& Evaluation, University of Toronto, Health Sciences Building, Toronto, ON, Canada, M5T 3M6 ${ }^{9}$ Department of Family Medicine, McMaster University, Hamilton, ON, Canada, L8S 4L8

${ }^{10}$ Department of Geriatric Medicine, University of Toronto, Toronto, ON, Canada, M5S $3 \mathrm{H} 2$

"Waterloo Wellington Local Health Integration Network, Waterloo, ON, Canada, N2J 2 A9

${ }^{12}$ Faculty of Science, Wilfrid Laurier University, Waterloo, ON, Canada, N2L 3C5

Background: Balance and strength training combined with adequate protein consumption may prevent falls and improve physical function in older adults. However, most older adults do not routinely engage in balance or strength training or consume enough protein. MoveStrong is a model to teach older adults about balance and functional strength training with attention to protein intake. The purpose of this study was to determine the association between MoveStrong and several outcomes.

Methods: MoveStrong is an eight-week pilot, assessorblinded, multisite, closed cohort stepped wedge randomized controlled trial. We recruited pre-frail or frail individuals $\geq 60$ years with at least one chronic condition who were not currently strength training. The program included sixteen exercise physiologist-led, one-hour group sessions on balance and functional strength training and two dietitianled one-hour sessions on protein intake. We conducted an exploratory analysis using linear regression adjusted for program exposure on responsiveness of frailty indicators, health-related quality of life, and physical activity levels at follow-up. We analyzed protein intake at baseline and followup using a generalized estimating equation.

Results: We enrolled 44 participants with an average age of $78 \pm 9.82$; 34 participants were pre-frail and nine, frail. We found a significant association between program exposure and the following variables: the 10-meter walk test $\left(F_{(2,173)}=21.776, p<0.001\right)$ with an $R^{2}$ of 0.201 , the 30 -second chair stand test $\left(F_{(2.173)}=11.291, p<0.001\right)$ with an $R^{2}$ of 0.115 , the four-square step test $\left(F_{(2,173)}=8.570\right.$, $p<0.001$ ) with an $R^{2}$ of 0.090 , physical activity levels $\left(F_{(1,174)}=11.555, p=0.001\right)$ with an $R^{2}$ of 0.062 , and self-rated health $\left(F_{(1,174)}=6.895, p=0.009\right)$ with an $R^{2}$ of 0.038 . There were no associations between MoveStrong and grip strength $\left(F_{(2,173)}=1.277, p=0.121\right)$, fatigue levels $\left(F_{(2.173)}=0.32, p=0.724\right)$, health-related quality of life $\left(F_{(1,174)}=2.750, p=0.009\right.$ ), or protein intake (mean change score: $2.90 \%, p=0.294$ ).

Conclusion: Exploratory analyses suggest that participating in the MoveStrong program is associated with improved gait speed, muscle strength and power, dynamic balance, physical activity levels, and self-rated health.

\section{SARCOPENIA AND VISION LOSS IN COMMUNITY-DWELLING OLDER PEOPLE}

Ya-Hsuan Tu, I-Jou Tan, Tsai-Ching Chiu, Kai-Yu Wu, Ching-Chieh Wang, Wen-Chun Lai, You-Syuan Lin, Shu-Chun Lee

School of Gerontology Health Management, College of Nursing, Taipei Medical University

Background: Visual impairment is common in older people. The most common causes of age-related visual impairment are presbyopia, cataracts, macular degeneration, and glaucoma, which affect visual acuity. Only two prior studies showed that visual impairment could be a predictor of low muscle mass and low muscle strength, indicating the potential correlation between visual impairment and 
sarcopenia. Therefore, the aim of study was to investigate the association between sarcopenia and vision loss.

Methods: A total of 100 community-dwelling older people was recruited and their sarcopenia was identified by the SARC-F questionnaire. Visual acuity was assessed by the smartphone-based visual acuity measurement and the values were represented in decimal. Level of visual acuity was categorized into normal visual function $(\geq 0.8)$, mild (0.3-0.8), and moderate to severe visual loss ( $<0.3$ ). Pearson correlation test was performed to measure the association between SARC-F score and visual acuity. Analysis of variance with post hoc Bonferroni test was conducted to compare the SARC-F scores among different levels of visual acuity.

Results: There was a significantly association between SARC-F score and visual acuity $(r=-0.348, p<0.001)$. Older people with moderate and severe visual loss had significantly higher SARC-F score than those with normal visual function $(-2.353,95 \% \mathrm{Cl}-4.695$ to $-0.011 ; \mathrm{p}=0.049)$ and mild vision loss $(-1.248,95 \% \mathrm{Cl}-2.379$ to $-0.117 ; \mathrm{p}=0.025)$. Conclusions: Sarcopenia is associated with vision loss in older people. Only when the visual acuity reaches a moderate level is there a significant relationship with sarcopenia. This may be due to the reduction of muscle function in visually impaired older people who restrict themselves from engaging in physical activities. Improvement of visual impairment, such as prescription glasses or surgical intervention, are needed in order to prevent sarcopenia. Older people with visual impairment should be advised to receive sarcopenia assessment.

\section{BIOMARKERS OF DIETARY INTAKE AND THEIR ASSOCIATION WITH PHYSICAL CAPABILITY ASSESSMENTS}

Thomas Wilson, David Langford, Laura Lyons, Manfred Beckmann, John Draper, Marco Arkesteijn

Institute of Biological Environmental and Rural Sciences, Aberystwyth University, Aberystwyth, United Kingdom

Background: Dietary intake and nutritional status is a key factor in the outcomes of older individuals. Changes in dietary habits, and nutritional intake contribute considerably to the progression of frailty and sarcopenia; especially in individuals who are already at risk of under-nutrition. Robust and highly acceptable home urine collection can provide objective dietary information, with minimal burden and can be used to detect shifts in dietary intake in individuals who may be transitioning into a pre-frail or frail state.

Methods: 38 individuals (male: 9, female: 29), aged over 60; collected three first morning void spot urine samples over the course of a week, immediately after attending physical capability screening. Standard physical capability measures were recorded for participants and body composition was measured using Dual-Energy X-ray absorptiometry (DXA). Urinary biomarkers were measured using liquid chromatography (LC) triple quadrupole mass spectrometry (MS), to give objective measures of habitual dietary intake.

Results: Pearson correlations between biomarker concentrations from a panel of 58 dietary biomarkers, showed that biomarkers of high protein containing foods (1-Methyl histidine, L-Carnosine, Taurine) were significantly positively associated with grip strength ( $r=0.36$ to 0.49 , $\mathrm{p}<0.05)$. L-Anserine and para-Cresol glucuronide were significantly associated with the number of chair rises in $\mathbf{3 0}$ seconds ( $r=0.33$ to $0.47, p<0.05$ ). For all physical capability assessments, elevated levels of biomarkers for high protein containing foods (in particular, high quality meat products) and protein catabolism, were associated with positive outcomes in the capability test.

Conclusions: This pilot study has demonstrated the feasibility of home urine collection in a group of older individuals and highlighted the association of dietary intake with physical capability. The use of urine biomarkers for low burden, objective measures of dietary intake has the potential for early detection of under-nutrition and declining physical capabilities.

\section{VISCERAL ADIPOSITY AND SKELETAL MUSCLE ARE IMPORTANT PROGNOSTIC INDICATORS IN LOCALLY ADVANCED RECTAL CANCER, INDEPENDENT OF BODY MASS INDEX}

\author{
Richard Gartrell ${ }^{1,2}$, Nicole Kiss ${ }^{4,5}$, Paul Baird ${ }^{6}$, \\ Justin Yeung 1,2,3 \\ 'Western Centre for Health Research and Education, Melbourne, \\ Australia \\ 2Department of Surgery, University of Melbourne, Melbourne, \\ Australia \\ ${ }^{3}$ Western Health Chronic Disease Alliance, Western Health, \\ Melbourne, Australia \\ IInstitute for Physical Activity and Nutrition, Deakin University, \\ Geelong, Australia \\ ${ }^{5}$ Allied Health Research, Peter MacCallum Cancer Centre, \\ Melbourne, Australia \\ ${ }^{6}$ Department of Surgery, Ophthalmology, University of Melbourne, \\ Victoria, Australia
}

Background: The prognostic significance of body composition and the association with BMI in locally advanced rectal cancer patients is not clear

Methods: We retrospectively identified 139 consecutive patients with stage 2-3 rectal cancer who underwent neoadjuvant chemoradiotherapy and surgery at Western Health, Victoria. Body composition was measured at the third lumbar vertebrae on pre-treatment imaging. Skeletal muscle index (SMI), adipose tissue and mean muscle attenuation (Hounsfield units) were determined. Primary outcomes were overall and disease-free survival. Univariate and multivariate analyses were conducted using Cox proportional hazard 
models. Linear regression modelling was used to assess the accuracy of the survival models.

Results: Among the 116 patients with pre-treatment scans, $50(43 \%)$ were sarcopenic and $63(54 \%)$ were viscerally obese. There was a wide variation of body composition across all BMl classes. Normal and underweight patients were more likely to be sarcopenic $(23 / 32,72 \%)$ than overweight and obese patients $(27 / 84,32 \%)(p<0.001)$ but less likely to be viscerally obese $(13 \%$ vs $70 \%$, $\mathrm{p}<0.001)$. Sarcopenia and BMI were not associated with survival outcomes. Elevated skeletal muscle index (HR 0.83, $95 \% \mathrm{Cl} 0.71-0.96, \mathrm{p}=0.015)$ and muscle attenuation (HR $0.84,95 \% \mathrm{Cl} 0.73-0.97, \mathrm{p}=0.017$ ) were associated with reduced mortality whilst visceral obesity, using pre-defined cut off points, was associated with worse overall survival (HR $6.43,95 \% \mathrm{Cl} 1.06-39.07, \mathrm{p}=0.043$ ) following multivariate analysis. Increased surface area of visceral (HR $1.16,95 \% \mathrm{Cl}$ $1.02-1.31, \mathrm{p}=0.019$ ) and subcutaneous (HR $1.17,95 \% \mathrm{Cl}$ $1.03-1.33 p=0.014$ ) adipose tissue were weakly negatively associated with overall and disease-free survival (HR 1.12, $95 \% \mathrm{Cl} 1.00-1.26 \mathrm{p}=0.046 ; \mathrm{HR} 1.13,95 \% \mathrm{Cl} 1.01-1.28$ $\mathrm{p}=0.036$ ).

Conclusions: Sarcopenia and visceral obesity are highly prevalent amongst an Australian population with locally advanced rectal cancer that is not accurately reflected by BMI. Visceral adiposity is negatively associated with overall and disease-free survival. Elevated SMI and muscle attenuation were protective for $\mathrm{OS}$.

\section{DISCOVERY SCIENCE ABSTRACTS}

\section{EFFECTS OF DIETARY RESTRICTION ON MUSCLE AGING IN CAENORHABDITIS ELEGANS}

Sobha Tumbapo, Adam Strudwick, Jana Stastna, Simon Harvey, Marieke Bloemink

Biomolecular Research Group, School of Human and Life Sciences, Canterbury Christ Church University, Canterbury CT 1 1QU, United Kingdom

Background: Sarcopenia leads to loss of functional mobility and independence in elderly people and is also a major component of frailty. With a growing aging population, it is essential to understand the molecular mechanisms behind sarcopenia in order to develop effective therapies.

Methods: In this study, GFP-labelled unc-54 (e 1092) strain of $C$. elegans were used to test the effect of three different dietary restriction (mild, medium and severe) on its lifespan and sarcopenia. This strain has one of its myosin heavy chain (MHC) isoform labelled with GFP so the fluorescence intensity can be used as an indicator of myosin density.

Results: Lifespan under mild and medium dietary restriction (DR) was significantly extended with a mean lifespan of 17 days in comparison to the control group (14 days). In contrast, the mean lifespan of worms under severe
DR decreased to 11 days in comparison to the control group. Worms under mild and medium DR also maintained significantly higher motility rates compared to severe DR and control group. Fluorescence measurements of the $C$. elegans body-wall muscle displayed an age-related decrease in fluorescence in all groups, consistent with reduced unc-54 myosin levels and indicating the development of sarcopenia. The fluorescence intensity in worms under mild and medium DR decreased at a slower rate compared to the severe DR and control group. Further analysis of the results revealed a proportional increase in healthspan with a decrese in gerospan (frailty period) for mild and medium DR worms while the opposite was true for worms under severe DR and control groups.

Conclusions: These findings suggest that moderately restricted diet is most effective in inducing the beneficial effects of DR on C. elegans lifespan and sarcopenia while severely restricted diet leads to detrimental effects.

\section{CAN RANKL INHIBITION AMELIORATE SARCOPENIA? AN IN VITRO STUDY}

John Gostage ${ }^{1,2}$, Katarzyna Goljanek-Whysall ${ }^{2,3}$, Ilaria Bellantuono', Eugene McCloskey'

'Department of Oncology and Metabolism, The Mellanby Centre For Bone Research, Healthy Lifespan Institute and The Centre for Integrated research in Musculoskeletal Ageing (CIMA). University of Sheffield, Sheffield, United Kingdom

${ }^{2}$ Discipline of Physiology, School of Medicine, National University of Ireland Galway, University Road, Galway, Ireland

3Institute of Ageing and Chronic Disease and The Centre for Integrated research in Musculoskeletal Ageing (CIMA). University of Liverpool, William Henry Duncan Building. 6 West Derby Street, Liverpool L7 8TX, UK

Background: The RANK/RANKL/OPG axis is critical in regulating osteoclastic bone resorption. Inhibition of RANKL by endogenous osteoprotegerin (OPG) or therapeutic denosumab inhibits osteoclast activation, reduces bone resorption, and prevents fractures. Placebo-controlled trials of denosumab for osteoporosis have also shown that it is associated with a significant reduction in falls, and in vivo mouse muscular dystrophy studies suggest protective effects of OPG on muscle function. This study seeks to determine if OPG effects the viability and differential capacity of primary mouse myoblasts/myotubes at different stages of in vitro myogenesis.

Methods: Non-toxic in vitro OPG doses and the effects of OPG on myoblast and myotube viability were determined by MTT. MF2O immunohistochemical staining was used to investigate phenotypic changes in differentiating myoblasts and myotubes treated with OPG.

Results: OPG $(5-20 \mathrm{ng} / \mathrm{ml})$ treatment was not cytotoxic to primary mouse myoblasts, differentiating myoblasts and myotubes. Interestingly, OPG significantly improved 
the viability of myotubes $(5 \mathrm{ng} / \mathrm{ml}: \mathrm{p}<0.05,10$ and $20 \mathrm{ng} /$ $\mathrm{ml}: \mathrm{p}<0.01$ ). OPG treatment to differentiating myoblasts positively modulated the myogenic phenotype, however a more prominent phenotype was observed when OPG was treated to myotubes. OPG significantly improved myotube phenotype, in a dose dependent manner, when treated at late stages of in vitro myogenesis. Most notably, myotube diameter ( $5 \mathrm{ng} / \mathrm{ml}: \mathrm{p}<0.01,10 \mathrm{ng} / \mathrm{ml}: \mathrm{p}<0.001)$ and fusion index (number of nuclei incorporated into myotubes; 5 and $10 \mathrm{ng} / \mathrm{ml}$ : $<<0.001$ ), were both significantly elevated.

Conclusions: OPG induced a hypertrophic myotube morphology suggesting OPG regulates myogenic activity in vitro, particularly at later stages of myogenesis (treatment of myotubes with OPG). These results suggest that RANKL inhibition may play a key role in regulating muscle mass and/or function. Future studies will explore if denosumab inhibition of human RANKL has effects on human myoblast/ myotube proliferation, differentiation, and regulation of myogenic factors to determine the potential for denosumab as a therapy for age-related muscle loss (sarcopenia).

\section{BNIP3 IS INVOLVED IN MUSCLE FIBER ATROPHY IN LATE-ONSET POMPE DISEASE}

Ana Carrasco-Rozas ${ }^{1}$, Esther Fernández-Simón ${ }^{1,2}$, Xavier Suarez-Calvet ${ }^{1,3}$, Patricia Piñol-Jurado ${ }^{1,2}$, Jorge Alonso-Pérez ${ }^{1}$, Benedikt Schoser ${ }^{4}$, Peter Meinke ${ }^{4}$, Isabel Illa ${ }^{1,3}$, Eduard Gallardo ${ }^{1,3}$, Jordi Díaz-Manera 1,2,3

'Neuromuscular Disorders Unit, Neurology Department, Hospital de la Santa Creu i Sant Pau and Biomedical Research Institute Sant Pau (IIB Sant Pau), Departament de Medicina, Universitat Autònoma de Barcelona. Spain

2John Walton Muscular Dystrophy Research Center, Newcastle University Translational and Clinical Research Institute, Newcastle Upon Tyne, UK

${ }^{3}$ Centro de Investigaciones Biomédicas en Red en Enfermedades Raras (CIBERER), Madrid, Spain

${ }^{4}$ Friedrich-Baur-Institute, Department of Neurology, LMU Klinikum, Munich, Germany

Background: Late-onset Pompe disease (LOPD) is a rare disorder produced by mutations in the GAA gene characterized by progressive muscle weakness. LOPD muscle biopsies show accumulation of glycogen inside lysosomes along with autophagic vacuoles associated with atrophic muscle fibers. The mechanism leading to muscle fiber atrophy and death is not well known. We have studied the role of molecules involved in the process of muscle fiber atrophy.

Methods: We analyzed the expression of several atrogens and their regulatory mechanisms on muscle biopsies ( $n: 7$ ) of LODP patients using RealTime-PCR, western blotting and immunofluorescence. We obtained myoblasts from biopsies of LOPD patients and studied their proliferation and differentiation rate. We transfected myoblasts with BNIP3 and studied size of the myotubes. We used non-parametric statistic studies to compare groups.
Results: We observed that muscle fibers containing vacuoles were smaller than fibers without vacuoles $(p<0.001)$. BNIP3 was expressed in all muscle fibers containing vacuoles, as demonstrated by immunofluorescence studies. BNIP3 positive fibers were smaller than negative fibers $(p<0.05)$. BNIP3 positive fibers were also positive for Ser317 phospho-ULK 1 demonstrating that mTOR pathway was inhibited in these fibers. Expression of BNIP3 did not induce apoptosis on muscle fibers but was associated with increases in cytochrome $\mathrm{C}$ sarcoplasm staining and expression of FUNDC1, suggesting that mitochondrial damage and mitophagy were active in these fibers. Transfection of BNIP3 to human myoblasts led to myotubes of smaller size than control myotubes suggesting their role in the process of muscle fiber atrophy.

Conclusion: These findings suggest a cascade with inhibition of the AKT-mTOR pathway, activation of expression of BNIP3 leading to mitochondrial damage, mitophagy, and finally to progressive muscle fiber atrophy. Our results open the door to potential new treatments targeting BNIP3 and reducing its deleterious effects on muscle fiber atrophy in Pompe disease.

\section{CIGARETTE SMOKING INDUCED DECREASE IN FIBRONECTIN AND COL 1 A 1 IN LOCOMOTOR MUSCLE IN A GUINEA PIG COPD MODEL}

Efpraxia Kritikaki ${ }^{1}$, Victor I Peinado², Joan A. Barbera ${ }^{3}$, Esther Barreiro ${ }^{4}$, Kay Padget ${ }^{1}$, Davina C.M. Simoes ${ }^{1}$

'Faculty of Health and Life Sciences, Northumbria University Newcastle, Newcastle Upon Tyne, UK

${ }^{2}$ Centro de Investigacio 'n en Red de Enfermedades Respiratorias, Instituto de Salud Carlos III, Bunyola, Majorca, Balearic Islands

3Servei de Pneumologia, Hospital Clinic, Institut d'Investigacions Biome diques August Pi i Sunyer, Universitat de Barcelona, Barcelona, Catalonia

${ }^{4}$ Pulmonology Department, Lung Cancer and Muscle Research Group, Hospital del Mar-IMIM, Parc de Salut Mar. Health and Experimental Sciences Department (CEXS), Universitat Pompeu Fabra (UPF), CIBERES, Barcelona, Spain

Background: Sarcopenia is a major comorbidity in patients with chronic obstructive pulmonary disease (COPD) caused by decreased physical activity capacity, cigarette smoke (CS) and chronic hypoxia $(\mathrm{CH})$. These risk factors affect muscle anabolic and catabolic biomarkers responsible for sarcopenia. However, whether $\mathrm{CS}$ and $\mathrm{CH}$ affect muscle extracellular matrix (ECM) biomarkers is still unknown. Intramuscular ECM plays an important role in muscle growth and repair processes, but their role in sarcopenia is under investigated. The aim of this study was to investigate alterations in the expression of intramuscular ECMs biomarkers in response to $\mathrm{CH}$ and $\mathrm{CS}$. We hypothesize that altered expression of the ECM molecules promoted by $\mathrm{CH}$ and $\mathrm{CS}$ contribute to development of sarcopenia. 
Methods: Skeletal muscle from a guinea pig animal model exposed (a) to 12-weeks CS (CS group), (b) to 10-week normal atmospheric air followed by 2-week of inspiratory oxygen fraction $(\mathrm{FI}, \mathrm{O2})$ of 0.12 in a hypoxic chamber $(\mathrm{CH}$ group), (c) to 12-week CS followed by 2-week $\mathrm{CH}$ (CS-CH group), (d) to 12-week sham-CS followed by 2-week normal atmospheric air (Control group) were analysed by Real-time PCR for the change in expression of intramuscular ECM biomarkers.

Results: Only CS treatment induced significant decreased in fibronectin and COLA 1 transcriptomes by $43 \%(p=0.05)$ and by $84.1 \%(p=0.026)$ respectively, compared to controls. $\mathrm{CH}$ and $\mathrm{CS}-\mathrm{CH}$ treatment downregulated fibronectin and COLA 1 , but not significantly compared to controls. No significant differences in ECM gene expression were observed when comparing $\mathrm{CH}$ with $\mathrm{CS}$ treatment.

Conclusion: CS affects ECM composition altering the expression of fibronectin and COL1A1. The role of ECM biomarkers to muscle sarcopenia is being investigated.

\section{INVESTIGATING THE ROLE OF CELLULAR SENESCENCE IN MUSCLE AGEING}

Leena Habiballa ${ }^{1,2,4}$, Richard Dodds ${ }^{1,2}$, Anthony Lagnado ${ }^{4}$, Xu Zhang ${ }^{4}$, Adam Hruby ${ }^{4}$, Mikolaj Ogrodnik ${ }^{4}$, Thomas von Zglinicki $^{3}$, James Kirkland ${ }^{4}$, Antoneta Granic ${ }^{1,2}$, Diana Jurk ${ }^{4}$, Avan Sayer ${ }^{1,2}$, Joao F. Passos ${ }^{4}$

'AGE Research Group, Translational and Clinical Research Institute, Faculty of Medical Sciences, Newcastle University, Newcastle upon Tyne, UK

${ }^{2}$ NIHR Newcastle Biomedical Research Centre, Newcastle upon Tyne Hospitals NHS Foundation Trust and Newcastle University, Campus for Ageing and Vitality, Newcastle upon Tyne, UK

${ }^{3}$ Institute for Cell and Molecular Biosciences, Newcastle University, Newcastle upon Tyne, UK

${ }^{4}$ Department of Physiology and Biomedical Engineering, Mayo Clinic, Rochester, MN, USA

Background: Sarcopenia is a major cause of loss of independence and reduced quality of life in older people. Cellular senescence refers to irreversible growth arrest and the production of a pro-inflammatory secretome in cells exposed to various stressors. Cumulative data has demonstrated that senescent cells accumulate with age at etiological sites in multiple tissues and that their elimination can alleviate several age-related pathologies in mice. The susceptibility of resident skeletal muscle cell populations to senesce with advancing age remains unclear and most studies on the subject rely on analyses of homogenised tissues, lacking the appropriate resolution to differentiate the relative contribution of specific cell-types.

Methods: In this study, we robustly characterised senescence in muscle during ageing and, importantly, identified specific cell-types responsible for the senescence burden. To establish a relationship of causality between senescence and muscle ageing phenotype, we cleared senescent cells using the INK-ATTAC mouse model. These investigations will also be replicated in the MASS_Lifecourse human tissue to determine the translational power of our findings.

Results: Our results indicate that senescence markers, including Telomere-associated DNA Damage, loss of HMGB 1 and LaminB1, SADS, and p21 increase significantly in myofibres and fibroadipogenic progenitor cells of mice with age. Importantly, we found a positive correlation between senescence markers and indicators of muscle quality, intramuscular fat accumulation, as well as blood cytokine levels. Clearance of senescent cells in aged mice using dasatinib and quercetin improve indicators of sarcopenia, in particular fibre size, fibrosis and fat accumulation compared to naturally aged mice and correlated with improved physical function. Current work involves investigating similar parameters in human muscle tissue.

Conclusions: Senescence markers increase significantly with age in muscle and correlate with severity of sarcopenia and intramuscular fat accumulation. Clearance of senescent cells improves indicators of sarcopenia, physical function, and intramuscular fat accumulation in old mice. Investigations into the contribution of senescence to muscle dysfunction in humans across the life course are currently underway.

\section{EPIDEMIOLOGY ABSTRACTS}

\section{WHAT IS THE ASSOCIATION BETWEEN} SARCOPENIA AND INCIDENT OSTEOPOROSIS? A PROSPECTIVE STUDY FROM UK BIOBANK

Fanny Petermann-Rocha',2, Lyn D. Ferguson ${ }^{2}$, Stuart R. Gray ${ }^{2}$, Irene Rodríguez-Gómez ${ }^{3,4}$, Naveed Sattar ${ }^{2}$, Stefan Siebert ${ }^{5}$, Frederick K. Ho ${ }^{1 *}$, Jill P. Pell' ${ }^{1}$, Carlos Celis-Morales ${ }^{2,6,7}$

'Institute of Health and Wellbeing, University of Glasgow, Glasgow, UK

${ }^{2}$ British Heart Foundation Glasgow Cardiovascular Research Centre, Institute of Cardiovascular and Medical Sciences, University of Glasgow, Glasgow, UK

${ }^{3}$ GENUD Toledo Research Group, Universidad de Castilla-La Mancha, Toledo, Spain.

${ }^{4}$ CIBER of Frailty and Healthy Aging (CIBERFES), Madrid, Spain.

5 Institute of Infection, Immunity \& Inflammation, University of Glasgow, Glasgow, UK

${ }^{6}$ Centre of Exercise Physiology Research (CIFE), Universidad Mayor, Santiago, Chile

'Laboratorio de Rendimiento Humano, Grupo de Estudio en Educación, Actividad Física y Salud (GEEAFyS), Universidad Católica del Maule, Talca, Chile

Background: The association of sarcopenia and incident osteoporosis has rarely been studied in prospective studies. This study aimed to investigate the association of sarcopenia 
and its components with incident osteoporosis in men and women from the UK Biobank study.

Methods: 168,684 participants ( $48.8 \%$ of women) were included in this prospective study. Sarcopenia (both presarcopenia and sarcopenia) and its individual components were defined according to the EWGSOP2 criteria (2019). Associations with incident osteoporosis were investigated using Cox-proportional hazard models adjusted for potential confounder factors: socio-demographic, lifestyle, healthrelated factors, and morbidity count.

Results: After a median follow-up of 7.4 years, 6,296 participants developed osteoporosis. After adjusting for relevant confounding factors, pre-sarcopenia was associated with 1.3 times higher risk of incident osteoporosis in men (HR: 1.30 [95\% Cl: 1.03 to 1.63]) but not in women. Sarcopenia was associated with 1.66 times increased incident osteoporosis risk in women (HR: 1.66 [95\% $\mathrm{Cl}: 1.33$ to 2.08 ]) but not in men compared with people without sarcopenia or pre-sarcopenia. In terms of individual components, low muscle mass ( $\mathrm{HR}_{\text {women }}: 1.36$ [95\% Cl: 1.17 to 1.51 ] and $\mathrm{HR}_{\text {men }}: 3.07$ [95\% Cl: 1.68 to 5.59 ]), followed by slow gait speed $\left(\mathrm{HR}_{\text {women }}: 1.30\right.$ [95\% Cl: 1.17 to 1.45$]$ and $\mathrm{HR}_{\text {men }}: 1.70$ [95\% Cl: 1.43 to 2.02]), were associated with a higher risk of incident osteoporosis in both sexes.

Conclusion: Pre-sarcopenic men and sarcopenic women had a higher risk of developing osteoporosis even after adjustment for a large range of potential confounders. Considering that sarcopenia could be prevented, health interventions to improve physical capability may delay or prevent the onset of osteoporosis.

\section{DIETARY OMEGA-3 POLYUNSATURATED FATTY ACID AND ALPHA-LINOLENIC ACID ARE ASSOCIATED WITH SARCOPENIA INDICES IN OLDER WOMEN 65-72 YEARS}

\author{
Masoud Isanejad ${ }^{1,2}$, Toni Rikkonen ${ }^{3}$, Behnam Tajik ${ }^{4}$, \\ Joonas Sirola ${ }^{4}$, Heikki Kröger ${ }^{4}$, Anne McArdle ${ }^{1}$, \\ Arja Erkkilä ${ }^{3}$ \\ 'Institute of Life Course and Medical Sciences, Department of \\ Musculoskeletal and Ageing Sciences, University of Liverpool \\ 2Liverpool Centre for Cardiovascular Science, University of \\ Liverpool and Liverpool Heart \& Chest Hospital, Liverpool, United \\ Kingdom \\ ${ }^{3}$ Kuopio Musculoskeletal Research Unit, University of Eastern \\ Finland, Kuopio Campus, Finland \\ ${ }^{4}$ Institute of Public Health and Clinical nutrition, University of \\ Eastern Finland, Kuopio Campus, Finland
}

Background: Association of dietary intake and serum level of polyunsaturated-fatty-acids (omega-3 PUFA) with physical function and body composition in older adults remains inconclusive. The aim was to investigate the association of dietary omega-3 PUFA (alpha-linolenic acid (ALA), eicosapentaenoic-acid (EPA), and docosahexaenoic- acid (DHA)) intake and serum omega-3 PUFA and DHA with physical function, muscle mass and fat mass in older women. Methods: Study subjects were 554 women from the Osteoporosis-Risk-Factor-and-Prevention-Fracture Prevention-Study, with dietary intake assessed with 3-day food record at baseline. Total omega-3 PUFA and DHA blood concentrations were analysed using a high-throughput proton (' $\mathrm{H})$ NMR spectrometry metabolomics platform at the University of Eastern Finland. Multiple physical function tests and short physical performance battery (SPPB) were calculated at the baseline and 3-year follow-up.

Results: The multivariable adjusted models showed statistically-significant associations for ALA with higher SPPB $(\beta=0.118, p=0.024)$, knee-extensionforce at baseline $(\beta=0.075, p=0.037)$ and lower fat mass $(\beta=-0.081, p=0.034)$, as well as longer oneleg-stance $(\beta$ baseline $=0.119, \quad \beta$ follow-up $=0.090$, $p=0.023$ ), higher walking speed ( $\beta$ baseline $=0.113$, $\beta$ follow-up $=0.144, p=0.047$ ), and ability to squat to the ground ( $\beta$ baseline $=0.110, \beta$ follow - up $=0.096$, $p=0.047$ ) at baseline and follow-up. Total omega-3 PUFA was statistically-significantly associated with better SPPB ( $\beta$ baseline $=0.108, p=0.039$ ), one-legstance $(\beta$ baseline $=0.102, p=0.041)$, walking speed $(\beta$ baseline $=0.110, \beta$ follow-up $=0.128, P=0.028$ ) and ability to squat ( $\beta$ baseline $=0.110, p=0.028$ ). Associations for dietary EPA and DHA, serum omega-3 PUFA and DHA with physical function and body composition were not significant.

Conclusion: Our results suggest that omega-3 PUFA and ALA may be important to SPPB and lower extremity function in older women. However, lack of association with serum concentration proposes that ALA and omega-3 PUFA may be bystander indicators of a healthy diet. Revealing the longterm effect of these nutrients with physical function requires further studies.

\section{CORRELATION BETWEEN SARCOPENIA AND TIMED UP AND GO, AND SARCOPENIA WITH PHYSICAL PERFORMANCE IN ELDERLY SUBJECTS IN SARIREJO VILLAGE, SEMARANG}

\section{Jimmy Tanamas', Yudo Murti Mupangati ${ }^{2}$}

'Internal Medicine Department, Faculty of Medicine, Diponegoro University/Dr. Kariadi Hospital Medical Center, Semarang, Indonesia ${ }^{2}$ Geriatric Division, Internal Medicine Department, Faculty of Medicine, Diponegoro University/Dr. Kariadi Hospital Medical Center, Semarang, Indonesia

Background: The population of older people in Indonesia is predicted to increase to 36 million in 2025. Increasing life expectancy will be accompanied by health problems, one of which is sarcopenia. Screening and diagnosis are required to identify sarcopenia especially in community-dwelling older people, so that early and comprehensive management can be applied. 
Methods: We conducted a cross-sectional study of an older population in Sarirejo village, Semarang, Indonesia. Participants were enrolled and information collected from a patient database. Mini-Cog assessment was performed to screen for cognitive impairment and nutritional status was assessed using the Mini Nutritional Assessment (MNA) score. Measurement of lower limb muscle mass was performed by dual x-ray absorptiometry (DXA; General Electric). Physical performance was assessed using the timed up and go (TUG) test. Participants were divided into three age groups based on the WHO classification ( $<=64$ years, $65-74$ years, $>=75$ years). Data were analyzed using descriptive statistics, with correlations calculated using Pearson and Spearman's correlations.

Results: 29 participants were enrolled; 27 (93.1\%) were women. 3 (10.3\%) had no education, $14(48.3 \%)$ had elementary level education, $4(13.8 \%)$ had junior high school level education, and $8(27.6 \%)$ had senior high school level education. 27 (93.1\%) were married, and $2(6.9 \%)$ were unmarried. The TUG score was positively associated with older age category ( $F=3.277, p=0.005)$, with older age correlated with longer TUG time $(r=0.477$, $p=0.009$ ). Higher $B M l$ was associated with higher lower limb muscle mass measured by DXA $(r=0.838, p<0.001)$. There was no significant correlation between the MNA score and sarcopenia measures $(p=0.419)$ or the TUG time with sarcopenia measures $(p=0.674)$.

Conclusion: There was no significant correlation between MNA score or physical performance measures and measures of sarcopenia in this population

\section{FOLLOWING THE STEPS OF THE EWGSOP2 TO DETECT SARCOPENIA ACCORDING TO AGE GROUPS IN OLDER WOMEN}

Anna Arnal-Gómez' ${ }^{1}$, Maria Àngels Cebrià i Iranzo ${ }^{1,2}$, Natalia Cezón-Serrano', Mercé Balasch-Bernat ${ }^{1}$, Maria Ángeles Tortosa-Chuliá ${ }^{3}$, Trinidad SentandreuMañó ${ }^{1}$, Silvia Forcano ${ }^{2}$

'Department of Physiotherapy, University of Valencia (Valencia, Spain)

${ }^{2}$ Hospital Universitari i Politècnic La Fe (Valencia, Spain)

${ }^{3}$ Department of Applied Economics, University of Valencia (Valencia, Spain)

Background: The European Working Group on Sarcopenia in Older People updated definition (EWGSOP2) follows different assessment steps and for each there are different measurements. Considering that aged population in Spain has a higher percentage of women, the aim of this study was to detect which measurement variables of the EWGSOP2 assessment steps decline in earlier ages in women in order to focus on them when identifying sarcopenia.

Methods: A multicenter cross-sectional study was carried out in older women ( $n=193$, mean age $77.8+/-9.1$ years), of the province of Valencia (Spain). The different assessment steps of the EWGSOP2 algorithm were analysed according to age groups (65-74, 75-84 and $\geq 85$ years): SARC-F, grip strength, chair stand, Appendicular Skeletal Muscle Mass (ASM), ASM Index, Short Physical Performance Battery (SPPB), gait speed, and Timed-Up-and-Go (TUG). Consequently, those variables which showed detection at earlier ages were included for the case finding and classification: 1) probable sarcopenia; 2) confirmed sarcopenia; and 3) severe sarcopenia. Statistical analysis included: Chi-square test and, 1-factor ANOVA or ANOVAWelch adjusted by Bonferroni or Games-Howell, respectively. Results: The chair stand is the only test that shows decline from 65 years old plus this continues being significant $(p<0.001)$ along the age groups. In the same way ASM, gait speed and SPPB start showing decline from 75 years old and it maintains ( $p<0.001$ ). On the contrary, ASM Index remains over the cut-off $\left(>5.5 \mathrm{~kg} / \mathrm{m}^{2}\right)$ in all age groups. Consequently, participants were classified according to chair stand, ASM and SPPB, showing that $50 \%$ of women aged $\geq 85$ had severe sarcopenia.

Conclusions: Chair stand, ASM and SPPB or gait speed seem to be early measurement detectors for older women. These measurements should be considered in clinical settings in order to apply early intervention.

\section{CLINICAL AND FUNCTIONAL CHARACTERISTICS OF SARCOPENIC VS NON SARCOPENIC OLDER WOMEN LIVING IN DIFFERENT SETTINGS}

Maria Àngels Cebrià i Iranzo 1,2, Natalia Cezón-Serrano', Anna Arnal-Gómez' ${ }^{1}$, Mercé Balasch-Bernat ${ }^{1}$, Maria Ángeles Tortosa-Chuliá ${ }^{3}$, Silvia Forcano², Trinidad Sentandreu-Mañó ${ }^{1}$

'Department of Physiotherapy, University of Valencia (Valencia, Spain)

${ }^{2}$ Hospital Universitari i Politècnic La Fe (Valencia, Spain).

${ }^{3}$ Department of Applied Economics, University of Valencia (Valencia, Spain)

Background: Sarcopenia is a prevalent condition with aging, and it is related to clinical and functional decline. Previously care setting has shown to play an important role in sarcopenia prevalence, plus in Spain the aged population has a high percentage of women. We aimed to analyse the clinical and functional characteristics of community-dwelling and institutionalised older women with or without sarcopenia.

Methods: A multicenter cross-sectional study was carried out in Valencia (Spain) including older women $(n=197$, mean age $77.7 \pm 8.9$ years), living in community $(n=95$, $48.2 \%)$ and in residential facilities $(n=102,51.8 \%)$. Sarcopenia participants were classified according to the European Working Group on Sarcopenia in Older People updated definition (EWGSOP2). Clinical variables measured 
were: number of medications and visits to doctor, number of hospitalisation stays and falls, and the abbreviated Charlson's Comorbidity Index; functional variables were: Barthel Index, quadriceps and biceps strength, and calf and arm circumferences. Statistical analysis included Student's t-tests for unpaired samples.

Results: Sarcopenic older women living in community took more medication $(p=0.021)$ and presented significant functional decline for Barthel Index and biceps strength (both $\mathrm{p}=0.0001$ ) compared to non-sarcopenic women. Institutionalised sarcopenic older women showed higher number of falls $(p=0.001)$, a decline of Barthel Index and biceps strength (both $p=0.0001$ ), and also a significant decline in quadriceps strength $(p=0.001)$ and calf circumference $(p=0.004)$ compared to non-sarcopenic women.

Conclusions: Number of medications and number of falls are characteristic of sarcopenic women living in community and institutionalised, respectively. The main functional variables that characterise sarcopenic women, whether they live in the community or institutionalised are Barthel Index and biceps strength. Clinicians may take these findings into consideration in order to identify sarcopenic women depending on their living setting.

\section{ASSOCIATION BETWEEN MILK INTAKE AND DECLINE IN MUSCLE STRENGTH FROM MID- TO LATE LIFE: THE MRC NATIONAL SURVEY OF HEALTH AND DEVELOPMENT}

Antoneta Granic ${ }^{1,2}$, Richard M Dodds ${ }^{1,2}$, Rachel Cooper ${ }^{3}$, Susan Hillman ${ }^{1,2}$, Avan A. Sayer ${ }^{1,2}$, Sian M. Robinson ${ }^{1,2}$

'AGE Research Group, Translational and Clinical Research Institute, Faculty of Medical Sciences, Newcastle University, Newcastle upon Tyne, United Kingdom

${ }^{2}$ NIHR Newcastle Biomedical Research Centre, Newcastle upon Tyne Hospitals NHS Foundation Trust and Newcastle University, Newcastle upon Tyne, United Kingdom

${ }^{3}$ Department of Sport and Exercise Sciences, Musculoskeletal Science and Sports Medicine Research Centre, Manchester Metropolitan University, Manchester, United Kingdom

Background: Cow's milk is a source of several essential nutrients which synergistically may be beneficial for skeletal muscle. Evidence that links milk intake to decline in muscle strength, a precursor of sarcopenia, from midlife to old age is lacking. We used data from the MRC National Survey of Health and Development to test whether mean total milk intake from age 36 to 60-64 years was associated with risk of probable sarcopenia (low grip strength, GS) and GS decline from age 53 to 69 years.

Methods: The analytic sample consisted of 2723 (50.8\% women) participants who had data for at least one exposure (milk) and one outcome (GS). Milk consumption was recorded in 5-day food diaries (ages 36, 43, 53 and 60-64 years), and grand mean of total, reduced-fat and full-fat milk each categorised in thirds ( $\mathrm{T} 1$ (lowest) to $\mathrm{T} 3$ (highest) consumption, $\mathrm{g} /$ day). Maximum GS was assessed at ages 53,60-64, and 69 years, and probable sarcopenia at age 69 defined by sex-specific cut-offs. We used logistic regression to examine the risk of probable sarcopenia, and multilevel models to investigate decline in GS in relation to milk intake thirds.

Results: Compared with T1, only T2 (58.7-145.2 g/day) of reduced-fat milk was associated with lower risk of probable sarcopenia at age 69 years (OR [95\% Cl]: 0.59 [0.37$0.94]$ ), after adjusting for key covariates at midlife, including social class, physical activity and BMI. The highest levels of consumption of total ( $\geq 237.5 \mathrm{~g} /$ day) and full-fat milk ( $\geq 107$ $\mathrm{g} /$ day) were associated with stronger GS in midlife in all participants ( $\beta(\mathrm{SE})=0.90(0.44), 95 \% \mathrm{Cl}$ : 0.04-1.77) and 0.89 (0.45), 0.01-1.77), respectively), but not with GS decline.

Conclusion: Data suggests that a higher milk consumption across adulthood may promote muscle strength in midlife and reduce risk of sarcopenia in later life.

\section{INVESTIGATING THE PREVALENCE OF SARCOPENIA, AND ITS ASSOCIATION WITH FRAILTY IN NORTHERN TANZANIA}

Samuel Bunn', Emma Grace Lewis ${ }^{1,2}$, Aloyce Kisoli ${ }^{3}$, Catherine Dotchin ${ }^{1,2}$, Richard Walker ${ }^{1,2}$

'Institute for Health and Society, Newcastle University

${ }^{2}$ Northumbria Healthcare NHS Foundation Trust

${ }^{3}$ Kilimanjaro Christian Medical Centre, Kilimanjaro Tanzania

Background: Sarcopenia has rarely been studied in older rural low-income country communities, yet may be an important contributor to poor health and impaired functioning. Bioimpedance analysis (BIA) and physical function tests used in the diagnosis of sarcopenia are associated with frailty. This study aimed to investigate the relationship between sarcopenia and frailty in rural northern Tanzania.

Methods: Data were analyzed from a randomly selected frailty-weighted sample of 235 adults aged $\geq 60$ years in northern Tanzania. These participants were selected from a screened population of 1207 older adults, representing the older population of five randomly selected villages in Hai district, Kilimanjaro region. A diagnosis of sarcopenia was derived using the European Working Group on Sarcopenia in Older People definition; using estimated appendicular skeletal muscle mass by BIA and grip strength. Those who also had poor physical performance by a low "timed up and go" (TUG) score were categorised as having severe sarcopenia. Frailty was identified through a comprehensive geriatric assessment.

Results: A strong association between frailty and sarcopenia was observed, $\mathrm{Chi}^{2}$ test $\mathrm{X}=35.475$ ( $\left.p<0.001\right)$, suggesting a relationship between the two syndromes. Of the 235 individuals, 194 had complete data for grip 
strength, anthropometric and BIA measurements, allowing a sarcopenia diagnosis to be derived. Forty seven (24.2\%) participants had sarcopenia, of whom 19 had severe sarcopenia. Adjusting for frailty-weighting, the estimated prevalence of sarcopenia and severe sarcopenia in the screened population was $19.5 \%$ and $5.4 \%$ respectively. Phase angle (by BIA), grip strength and TUG all predicted frailty independently in multivariate regression analyses.

Conclusion: The estimated prevalence of sarcopenia in this study is higher than the overall global estimated prevalence of $10 \%$ for adults $\geq 60$ years. This high burden of sarcopenia is likely to be an important contributor to poorer health and functioning for older people in this setting.

\section{THE PREVALENCE OF SARCOPENIA IN PARKINSON'S DISEASE AND RELATED DISORDERS}

Ashley Hart', ${ }^{1,2}$ Fred Barker', Avan A Sayer ${ }^{1,3}$,
Antoneta Granic', Alison Yarnall,2,
'AGE Research Group, NIHR Newcastle Biomedical Research
Centre, Newcastle University and Newcastle Upon Tyne Hospitals
NHS Foundation Trust, Newcastle, UK
${ }^{2}$ Brain and Movement Research Group, Translational and Clinical
Research Institute, Campus for Ageing and Vitality, Newcastle
University UK
${ }^{3}$ Newcastle upon Tyne NHS Foundation Trust, Newcastle, UK

Background: There is evidence that sarcopenia is more prevalent in Parkinson's disease (PD) than in age matched cohorts. Shared features of pathogenesis include IL-6 driven neuroinflammation and mitochondrial dysfunction. This study systematically reviews the prevalence of sarcopenia in PD and atypical Parkinsonian syndromes (aPS), how prevalence differs by PD disease stage and between PD and aPS.

Methods: Predefined literature search strategies were used on MEDLINE, EMBASE, Scopus and Web of Science. Studies conducted in people with PD or aPS reporting on the prevalence of sarcopenia or those that provided enough data to compute this using validated sarcopenia definitions were included. Risk of bias was assessed using the AXIS tool.

Results: 1683 (199 duplicates) studies were identified through the search; 67 were assessed in full and 14 met inclusion criteria. 11 gave estimates of sarcopenia and 3 probable sarcopenia. The average score for study quality was 14.5/20 (range 11-18). The mean prevalence of sarcopenia in Parkinsonian patients was $17.9 \%$ (range 2-31.4\%). The mean prevalence of probable sarcopenia (i.e. low muscle strength) was $49.1 \%$ (range $17.5-76.7 \%$ ). Across all studies reporting on non-Parkinsonian controls, sarcopenia was more prevalent in those with Parkinsonian disorders. The combined odds ratio was 2.49 ( $p<0.0001$ ). Six studies reported a mean UPDRS III score. Mean UPDRS III score positively correlated with the prevalence of sarcopenia though this finding was not consistently reflected in individual studies. Three studies commented on the prevalence of sarcopenia in aPS. There was little agreement between studies as to whether sarcopenia is more prevalent in PD or aPS.

Conclusions: Sarcopenia is a prevalent and important issue in Parkinsonian disorders. Probable sarcopenia is especially common. Recognition of this should prompt clinical testing and provision of appropriate interventions to minimise negative clinical outcomes associated with movement disorders being compounded by muscle weakness.

\section{ADIPOSITY AND GRIP STRENGTH: A MENDELIAN RANDOMISATION STUDY IN UK BIOBANK}

Snehal M. Pinto Pereira ${ }^{1}$, Victoria Garfield ${ }^{2}$, Aliki-Eleni Farmaki ${ }^{2}$, David J. Tomlinson ${ }^{3}$, Ghazaleh Fatemifar ${ }^{4}$, Spiros Denaxas ${ }^{4}$, Chris Finan ${ }^{5}$, Rachel Cooper ${ }^{3}$

'Institute of Sport, Exercise and Health, Division of Surgery \& Interventional Science, University College London, London, UK ${ }^{2}$ MRC Unit for Lifelong Health \& Ageing, Institute of Cardiovascular Science, University College London, London, UK

${ }^{3}$ Department of Sport and Exercise Sciences, Musculoskeletal Science and Sports Medicine Research Centre, Manchester Metropolitan University, Manchester, UK

${ }^{4}$ Institute of Health Informatics, University College London, London, UK

5Institute of Cardiovascular Science, University College London, London, UK

Background: Muscle weakness at older ages is a major public health concern. Grip strength is commonly used to identify weakness and an improved understanding of its causal determinants is required. We aimed to establish associations between total and central adiposity and grip strength.

Methods: Up to 470,786 UK Biobank participants with data on four indicators of adiposity (body mass index (BMI), body fat percentage (BF\%), waist circumference (WC) and waist-hip-ratio (WHR)) and maximum grip strength were included. Using linear regression, we examined sex-specific associations between each adiposity indicator and grip strength. We explored whether associations varied by age, by stratifying analyses by age-group ( $<50 y, 50-59 y, 60-64 y$, $\left.65 y^{+}\right)$. Using Mendelian Randomisation (MR), we estimated the strength of the same associations using genetic instruments for each adiposity trait as our exposures.

Results: In men, observed and MR associations were generally consistent: higher BMI and WC and lower WHR were associated with stronger grip: in covariate adjusted observational analyses, 1-SD higher BMI was associated with $0.75 \mathrm{~kg}$ ( $95 \% \mathrm{Cl}: 0.71 \mathrm{~kg}, 0.79 \mathrm{~kg})$ stronger grip; $1-\mathrm{SD}$ higher WHR was associated with $0.23 \mathrm{~kg}(95 \% \mathrm{Cl}: 0.19$ $\mathrm{kg}, 0.27 \mathrm{~kg}$ ) weaker grip. Associations of BMI and WC with 
strength attenuated with age: in men aged $<50 y$ and $65 y+$, 1 -SD higher BMI was associated with $1.16 \mathrm{~kg}(95 \% \mathrm{Cl}$ : $1.08 \mathrm{~kg}, 1.25 \mathrm{~kg})$ and $0.37 \mathrm{~kg}(95 \% \mathrm{Cl}: 0.28 \mathrm{~kg}, 0.46 \mathrm{~kg})$ stronger grip, respectively. In women, $\mathrm{BF} \%$ associations with grip strength varied by age; associations for BMI, WC and WHR remained broadly stable over age-groups. Only BF\% associations were directionally consistent in observational and MR analyses.

Conclusions: Using different methods to triangulate evidence on the effects of adiposity on grip strength, we found consistent associations of higher BMl and WC and lower WHR with stronger grip in men; findings in women were less consistent. We strengthen the evidence base for causal links between adiposity and grip strength in men, whereas links between adiposity and grip strength in women are less clear.

\section{DYNAPENIC ABDOMINAL OBESITY AS A RISK FACTOR FOR FALLS AND FALL-RELATED INJURIES}

Lisa Dowling ${ }^{1}$, D.J. Cuthbertson ${ }^{2}$, Jennifer Walsh ${ }^{1}$

'Department of Oncology and Metabolism, University of Sheffield, Sheffield, UK

2Institute of Ageing and Chronic Disease, University of Liverpool, Liverpool, UK

Background: Dynapenia, or low muscle strength, and obesity are independently associated with a greater risk of falling. This observational cohort study aimed to determine whether Dynapenic Abdominal Obesity (DAO) conferred a greater risk of falls and fall-related injury than either dynapenia or obesity alone.

Methods: In 4239 participants (60-90 years) from the English Longitudinal Study of Ageing, hand-grip strength (HGS), sit-to-stand time (STS), gait speed, body mass index (BMI) and waist circumference (WC) were measured at Wave 6. Low muscle strength, or dynapenia, was defined according to European Working Group on Sarcopenia in Older People (EWGSOP), EWGSOP2 and the Sarcopenia Definitions and Outcomes Consortium (SDOC) criteria. Data on falls and fallrelated injuries (2-year follow-up) were collected at Wave 7. Multiple logistic regression analyses were performed adjusting for age, sex and previous fall history. Area under the receiver operating characteristic curve (AUC) was calculated from logistic regression models.

Results: Falls occurred in 1049 participants, of whom 284 reported an injury during follow-up. High BMI ( $1.21,1.04-$ 1.42 p=0.015; Odds Ratio, 95\% Confidence Interval), low HGS according to EWGSOP criteria $(1.32,1.10-1.60$ $\mathrm{p}=0.003)$ and poor STS (1.49, 1.22-1.88 $\mathrm{p}<0.001)$ were individually associated with falling. Greater WC and either poor STS or EWGSOP-HGS had additive effects on falls risk. Individual discrimination was low for measures of obesity or dynapenia either alone or in combination (AUC 0.51-0.58). There was no relationship between fall- related injuries and obesity or dynapenia ( $p>0.05)$. Conclusion: Abdominal obesity and dynapenia according to EWGSOP criteria were independently associated with falls. Our findings support the hypothesis of a cumulative risk associated with DAO on falls risk. No association was found between obesity and dynapenia and fall-related injury.

\section{LONG-TERM CONDITIONS, MULTIMORBIDITY, LIFESTYLE FACTORS AND CHANGE IN GRIP STRENGTH OVER 9 YEARS OF FOLLOW- UP: FINDINGS FROM 44,3 15 UK BIOBANK PARTICIPANTS}

Christopher Hurst ${ }^{1,2}$, James Murray ${ }^{1,2}$, Antoneta Granic ${ }^{1,2}$, Susan J. Hillman ${ }^{1,2}$, Rachel Cooper ${ }^{3}$, Avan A. Sayer ${ }^{1,2}$, Sian M. Robinson ${ }^{1,2}$, Richard M. Dodds ${ }^{1,2}$

'AGE Research Group, Translational and Clinical Research Institute, Faculty of Medical Sciences, Newcastle University, Newcastle, UK

${ }^{2}$ NIHR Newcastle Biomedical Research Centre, Newcastle University and Newcastle upon Tyne NHS Foundation Trust ${ }^{3}$ Department of Sport and Exercise Sciences, Musculoskeletal Science and Sports Medicine Research Centre, Manchester Metropolitan University, Manchester, UK

Background: Weak grip strength is associated with a range of adverse health outcomes, and an accelerated decline in grip strength confers an even greater risk. To date there has been limited research into the factors associated with change in grip strength in mid-life. Using data from UK Biobank our aim was to investigate the associations of long-term conditions (LTCs), multimorbidity and lifestyle factors with patterns of change in grip strength.

Methods: We used data from 44,3 15UK Biobank participants with grip strength measured at baseline (2006-10) and a subsequent study visit around nine years later. At baseline, participants' LTCs were self-reported and categorised against a hierarchy. Multimorbidity was characterised by the number of LTC categories present ( $0,1,2$ and $3+$ ). Lifestyle factors (body mass index (BMI), smoking, diet and physical activity) were also assessed. Change in grip strength was grouped into four patterns: decline, stable low, stable high or reference (no change or increase) and used as the outcome in multinomial logistic regression models.

Results: Most LTC categories were associated with adverse patterns of change in grip strength (stable low and/or decline): for example, musculoskeletal/trauma conditions were associated with an increased risk of the stable low pattern (Relative Risk Ratio [RRR]=1.63; 95\% Confidence Interval [Cl] 1.49-1.79). Multimorbidity and lifestyle factors had independent associations with grip strength change: those with 3+ categories of LTCs were more likely to experience decline in grip strength (RRR $=1.18 ; 95 \% \mathrm{Cl} 1.08-1.28$ ) compared to those with no LTCs. Low physical activity was associated with adverse patterns of grip strength, while raised BMI had divergent associations (both the decline and 
stable high patterns of grip strength change).

Conclusions: Individuals living with multimorbidity and those with lifestyle risk factors such as low physical activity are at increased risk of loss of strength over time.

\section{PHYSICAL FRAILTY COMPONENTS AND RISK PREDICTION OF COVID-19 OUTCOMES: UK BIOBANK ANALYSIS}

Monika Mickute ${ }^{1,2}$, Francesco Zaccardi ${ }^{1,3}$, Cameron Razieh ${ }^{1,2}$, Jack Sargeant ${ }^{1,2}$, David Webb ${ }^{1,2}$, Thomas J. Wilkinson ${ }^{2,4}$, Alice C. Smith ${ }^{2,4}$,

Alex V. Rowlands ${ }^{1,2}$, Clare Gillies ${ }^{1,3}$, Yogini Chudasama ${ }^{3}$, Claire Lawson ${ }^{1,3}$, Tom Norris ${ }^{5}$, Melanie J. Davies ${ }^{1,2}$, Kamlesh Khunti ${ }^{1,2,6}$, Thomas Yates ${ }^{1,2}$

'Diabetes Research Centre, University of Leicester, Leicester General Hospital, Leicester, LE5 4PW, UK

${ }^{2}$ National Institute for Health Research (NIHR) Leicester Biomedical Research Centre (BRC), NIHR Leicester Biomedical Research Centre, University of Leicester, Leicester, UK

${ }^{3}$ Leicester Real World Evidence Unit, Diabetes Research Centre, University of Leicester, Leicester, LE5 4PW UK

${ }^{4}$ Leicester Kidney Lifestyle Team, Department of Health Sciences, University of Leicester, Leicester, UK

${ }^{5}$ School of Sport, Exercise and Health Sciences, Loughborough University, UK

${ }^{6}$ NIHR Applied Research Collaboration - East Midlands (ARC-EM), University of Leicester, Leicester, UK

Background: Physical frailty has been shown to be associated with severe COVID-19 infection. This study aimed to assess the predictive discrimination of each physical frailty component and overall frailty status in predicting COVID-19 outcomes.

Methods: This report included Biobank participants recruited between 2006-2010 with COVID-19 test data. C-index was used to examine predictive discrimination of individual Fried's frailty components (including slowness, weakness, low physical activity, exhaustion, weight loss) and overall frailty status for two COVID-19 outcomes: 1) Mild disease: a positive COVID- 19 test from a community setting without evidence of severe disease 2) Severe disease: a positive inhospital COVID-19 test or COVID-19 as a primary cause of death defined as severe COVID-19.

Results: Out of 358,433 (54.5\% females, median age 68 years) included in the main analysis, 9,679 patients were classified as having mild COVID-19 and 2,964 were classified as having severe COVID-19. The prevalence of physical frailty was highest in the severe COVID-19 group (8.1\%) compared to mild COVID-19 group (3.2\%) and with no COVID-19 diagnosis (2.9\%). For severe COVID-19 outcomes, self-reported walking pace provided the greatest risk discrimination compared to other individual frailty indicators, or overall frailty status. Adding walking pace to a base model containing age and sex improved $\mathrm{C}$-index by $0.039(95 \% \mathrm{Cl} 0.031,0.046)$ and by $0.005(95 \% \mathrm{Cl}$
$0.003,0.008$ ) when added to a model containing age, sex, material deprivation, comorbidities and body mass index. Results for mild COVID-19 were similar, however C-index improvement were lower.

Conclusions: A simple self-reported measure of walking pace provides the greatest predictive discrimination for severe COVID-19 outcome compared to other frailty indicators or overall frailty status.

\section{PROTEIN AND MICRONUTRIENT INTAKES IN ELDERLY LIVING IN NURSING HOMES IN NORTHEAST SPAIN: ENOUGH TO PREVENT SARCOPENIA?}

\section{N. Piedrafita, M.A. Romero-Rodríguez,}

M.L. Vásquez-Odériz

Universidad Santiago Compostela, Departamento de Química Analítica, Nutrición y Bromatología. Facultad de Ciencias. Campus de Lugo. Galicia. Spain

Background: Sarcopenia is associated with a low intake of energy and nutrients. The nutrients that have been most consistently linked to the components of sarcopenia in observational studies include protein, vitamin $D$ and antioxidant nutrients (that include carotenoids, selenium, magnesium, calcium and vitamins $\mathrm{E}$ and $\mathrm{C}$ ), which appear to influence muscle mass and function.

Methods: This research was part of a cross-sectional study called "Nutriage project", which included a sample of permanent residents in fifteen nursing homes for older adults in Galicia (Northeast Spain). Food intake data were collected by the precise weighing method. Results were compared with Dietary Reference Intakes for >70-yearolds, alongside recommendations of the European Society for Clinical Nutrition and Metabolism and PROT-AGE group. The variables analyzed were energy, protein, antioxidant nutrients and vitamin $D$ in both sexes. Means for nutrient intake were calculated, and differences by sex and age categories were tested using $t$-test or ANOVA. $P$ values $<0.05$ were considered statistically significant.

Results: The mean energy intake was $1830 \pm 434$ kcal. Women consumed $11 \%(183 \mathrm{kcal})$ more energy than recommended. The mean daily intake of protein was $1.2 \pm 0.5 \mathrm{~g} / \mathrm{kg}$ of BW. The mean intake of selenium, calcium, magnesium, vitamin $\mathrm{D}, \mathrm{C}$ and $\mathrm{E}$ was $130 \pm 73 \mathrm{mg} /$ day; $1068 \pm 178 \mathrm{mg} /$ day; $256 \pm 71 \mathrm{mg} /$ day; $1.4 \pm 0.9 \mathrm{mcg} /$ day; $146 \pm 106 \mathrm{mg} /$ day and $3 \pm 2 \mathrm{mg} /$ day, respectively. No significant differences were found between the sexes ( $p>0.05$ ).

Conclusions: The dietary intake of the institutionalized elderly in this study did not meet the nutritional requirements recommended in terms of calcium, magnesium, vitamin $D$ and $E$. Based on these findings, the nutritional intake of nursing homes is inadequate, especially taking into account the published scientific evidence on the contribution of proteins and specific nutrients for the prevention of sarcopenia. 


\section{CLINICAL PRACTICE ABSTRACTS}

\section{AWARENESS REGARDING FRAILTY AND SARCOPENIA AMONG HEALTHCARE PROFESSIONALS AT DISTRICT GENERAL HOSPITAL IN THE SOUTH EAST OF THE UNITED KINGDOM}

Clarence M.P. Chikusu, Joel James, Amritha Narayanan Ashford and St Peter's NHS Foundation Trust

Background: Frailty and muscle strength are critical components of walking ability and the presence of these can result in a high prevalence of falls. They also result in increased morbidity and mortality among the elderly. Despite sarcopenia being very common and a reversible condition in its early stage it is a frequently overlooked and undertreated geriatric syndrome. A greater understanding of sarcopenia and frailty among healthcare professionals could have a dramatic impact on outcome and quality of life of the elderly. Objectives: This study aimed to assess the current knowledge about the concept of sarcopenia and frailty among the healthcare professionals working in an NHS District General Hospital in Surrey, UK.

Methods: This longitudinal study included NHS healthcare professionals $(n=50)$ who were asked to complete a questionnaire regarding awareness of concepts, risks, diagnostic strategy and management of frailty and sarcopenia.

Results: $63.27 \%$ of healthcare professionals stated to know the concept of sarcopenia, $20 \%$ indicated that they knew how to diagnose sarcopenia and $20 \%$ had seen patients with suspected sarcopenia in the last one month. Only $20 \%$ knew the risk associated with sarcopenia. 83.33\% used the SARC-F questionnaire as diagnostic method for sarcopenia. $100 \%$ of the cohort experienced bottlenecks during the implementation of diagnostic strategy. Lack of awareness and time (41.76\%) was the main reason for this. $97.96 \%$ knew the term frailty and $76.16 \%$ knew that sarcopenia and frailty were not the same. $77.55 \%$ were aware of the scoring methods for frailty and $76.32 \%$ used clinical frailty score as the method. $65.31 \%$ was aware of the frailty pathway but only $53.06 \%$ knew whom to contact regarding managing frailty. $57.14 \% \mathrm{knew}$ the term comprehensive geriatric assessment. Only $24.49 \%$ was aware of key recommendations of managing frailty in the acute setting.

Conclusion: Although concepts of sarcopenia and frailty are familiar to most NHS healthcare professionals, the practical and clinical application is limited due to a lack of awareness regarding the diagnostic methodology, risks as well as time constrains. As such the benefits and potential treatment options may be overlooked and we aim to improve awareness so that these measures can improve outcomes for patients.

\section{PROTEIN INTERVENTIONS AUGMENT THE EFFECT OF RESISTANCE EXERCISE ON LEAN MASS AND STRENGTH IN OLDER ADULTS: A SYSTEMATIC REVIEW AND META-ANALYSIS OF RANDOMIZED CONTROLLED TRIALS}

Richard Kirwan', Carmen Rodríguez García², Mohsen Mazidi ${ }^{3}$, Katie Lane ${ }^{4}$, Tom Butler ${ }^{5}$, Fatima Perez de Heredia', Ian G. Davies ${ }^{4}$

'School of Biological and Environmental Sciences, Liverpool John Moores University, Liverpool, UK

${ }^{2}$ Department of Health Sciences, Faculty of Experimental Sciences, University of Jaén, 23071 Jaén, Spain

${ }^{3}$ Department of Twin Research and Genetic Epidemiology, King's College London, London, UK

${ }^{4}$ Research Institute of Sport and Exercise Science, Liverpool John Moores University, Liverpool, UK

${ }^{5}$ School of Applied Health and Social Care and Social Work, Faculty of Health, Social Care and Medicine, Edge Hill University, UK

Background: Increased protein intake is suggested as a strategy to slow or even reverse the age-associated loss of muscle mass and strength observed in sarcopenia. However, the diversity of study designs results in inconsistent findings on the effects of protein interventions in older adults.

Methods: We assessed the evidence on the effects of whole protein supplementation or higher-protein diets, without the use of essential amino acids or supplements known to stimulate hypertrophy, alone or in combination with resistance exercise interventions, on lean body mass and strength in older adults. We performed a systematic literature search using PubMed, Medline, Web of Science and Cochrane CENTRAL databases from January 1990 up to October 2019. We included randomized controlled trials assessing whole protein supplementation and/or higherprotein dietary interventions in older adults (mean age $\geq 50$ years), on total lean body mass, appendicular lean mass, hand grip and knee extension strength. We used a random effects model (weighted mean difference [WMD]) and generic inverse variance methods were used to synthesize quantitative data, followed by a leave-one-out method for sensitivity analysis.

Results: Protein supplementation had a significant positive effect on total lean body mass [WMD: $0.247, p=0.006, l^{2}$ : $14.2 \%$ ], appendicular lean mass [WMD: $0.551, p=0.018, l^{2}$ : $0 \%$ ] and knee extension strength [WMD: 0.435, $\mathrm{p}=0.026$, $I^{2}:$ 63.9\%]; however, only when used concomitantly with resistance exercise. Neither protein nor resistance exercise intervention had significant effects on handgrip strength [WMD: $0.126, p=0.141, I^{2}$ : 0\%]. Meta-regression revealed significant positive associations between baseline total lean body mass and change in total lean body mass (beta: $0.0034, p=0.013$ ) and between per-meal protein dose and change in appendicular lean mass (beta: $0.016, p=0.041$ ). Conclusions: In older adults undergoing resistance exercise, increased protein intake leads to greater lean body mass, 
appendicular lean mass and knee extension strength, although no superior effect is seen with the use of additional protein alone.

\section{VALIDITY OF THE SARQOL QUALITY OF LIFE TOOL IN A UK POPULATION OF OLDER PEOPLE WITH IMPAIRED PHYSICAL FUNCTION: FINDINGS FROM SarcNet}

Philip Heslop', Richard Dodds ${ }^{1}$, Andrew Clegg², Suzy Hope ${ }^{3}$, Claire McDonald ${ }^{4}$, David Smithard ${ }^{5}$, Bryony Storey ${ }^{4}$, Ai Lyn Tan ${ }^{6}$, Anna Thornhill?, Avan Sayer' ${ }^{1}$ Miles Witham ${ }^{1}$

'AGE Research Group, NIHR Newcastle Biomedical Research Centre, Newcastle University and Newcastle upon Tyne Hospitals NHS Foundation Trust, Newcastle, UK

${ }^{2}$ Academic Unit for Ageing and Stroke Research, University of Leeds, Bradford Teaching Hospitals NHS Foundation Trust, Bradford, UK

${ }^{3}$ College of Medicine and Health, University of Exeter, and Royal Devon \& Exeter NHS Foundation Trust, Exeter, UK

${ }^{4}$ Department of Geriatrics, Gateshead Health NHS Foundation Trust, Gateshead, UK

${ }^{5}$ Queen Elizabeth Hospital, Lewisham and Greenwich NHS Trust and University of Greenwich, London, UK

${ }^{6}$ NIHR Leeds Biomedical Research Centre, Chapel Allerton Hospital, and Leeds Institute of Rheumatic and Musculoskeletal Medicine, University of Leeds, Leeds, UK

${ }^{7}$ Academy of Research and Improvement, Solent NHS Trust, Portsmouth, UK

Background: SarQoL is a disease-specific quality-of-life tool for use by patients with sarcopenia. Comprehensive validation data for SarQoL in a UK population with sarcopenia are lacking. This study tests SarQol's internal consistency, reliability, responsiveness and external validity in a UK sarcopenia cohort.

Methods: Data from the SarcNet pilot sarcopenia registry study were analysed. Participants were aged $>=65$ with a SARC-F score $>=3$, recruited from primary care practices. SarQoL was administered at baseline and six-month followup. Participants self-assessed six-month global change in a) overall fitness, and b) overall quality of life on a 7-point Likert scale ('much worse' to 'much better'). Internal consistency of SarQoL and its subdomains was analysed using Cronbach's alpha. Reliability was analysed using intra-class correlation coefficients (ICC) for participants reporting 'no change' in global status. Cohen's d and Guyatt's responsiveness coefficients were calculated separately for groups reporting 'slight improvement', 'slight worsening', or any improvement. External (criterion) validity was assessed via correlation between baseline SarQoL, physical performance measures, and SARC-F score.

Results: We analysed data from 147 participants at baseline, and 125 at follow-up. SarQoL had overall excellent internal consistency (Cronbach's alpha 0.944 [95\% Cl 0.930,
0.956]) at baseline, but modest at follow-up (0.7 10 [95\% $\mathrm{Cl} 0.632,0.778]$ ). Consistency within most subdomains was good except 'body composition' and 'leisure activities'. Reliability was moderate (ICC 0.59 to 0.66 ). Responsiveness to change was greater for those reporting global improvement than for those reporting global deterioration (Cohen's $\mathrm{d} 0.03$ to 0.36 for deterioration; 0.75 to 2.29 for improvement). Baseline SarQoL showed moderate correlation with SARC-F $(r=-0.45, p<0.001)$, grip strength $(r=0.37, p<0.001)$, Short Physical Performance Battery $(r=0.48, p<0.001)$ and gait speed $(r=0.48, p<0.001)$.

Conclusion: SarQoL had good internal consistency, moderate reliability, and evidence of criterion validity in cross-sectional comparison with related measures.

\section{IMPROVING EXERCISE INTERVENTIONS FOR OLDER PEOPLE LIVING WITH SARCOPENIA AND FRAILTY THROUGH A NEW UK-WIDE BENCHMARKING AND FEEDBACK PROJECT}

Lorna Caulfield ${ }^{1}$, Charlotte Buckland ${ }^{2}$, Melody Chawner ${ }^{3}$, Sarah De Biase', Philip Heslop' ${ }^{1}$, Christopher Hurst ${ }^{1}$, Avan Sayer ${ }^{1}$, Miles Witham ${ }^{1}$

'AGE Research Group, NIHR Newcastle Biomedical Research Centre, Newcastle University and Newcastle upon Tyne Hospitals NHS Foundation Trust, Newcastle, UK

${ }^{2}$ Newcastle upon Tyne Hospitals NHS Foundation Trust, Newcastle, UK

${ }^{3}$ Solent NHS Trust, Portsmouth, UK

${ }^{4}$ AGILE, Professional Network recognised by Chartered Society of Physiotherapy, London, UK

Background: A growing body of evidence supports resistance training as a safe and effective intervention for older persons at risk of, or living with, sarcopenia and frailty. Recent surveys of current UK practice in exercise interventions for older people with sarcopenia and frailty found that resistance training was offered in only $9 \%$ of departments, and exercise programmes are often not optimized for sarcopenia and frailty.

Methods: This initiative will develop a benchmarking and feedback system to identify and promote aspects of good practice in exercise training for older people with sarcopenia and frailty. The project will invite physiotherapists and exercise practitioners across the UK who are involved in the delivery of exercise interventions for sarcopenia and frailty to take part. Practitioners will be asked to upload anonymized information on twenty consecutive patients undertaking exercise training for sarcopenia and/or frailty using an online data collection portal. We are seeking at least twenty-five practitioners to take part. Alongside anonymized baseline details, data will be collected on the structure of intervention pathways; reasons for referral; intervention characteristics including modalities of training and the frequency, duration and intensity of the interventions; progression of exercise 
interventions; and patient outcomes, including the methods used to assess these.

Analysis and Projected Outcomes: The data will be reviewed by an expert panel to identify which characteristics of exercise programmes are associated with the best outcomes. This information will contribute to a series of recommendations for best practice. Individual participant practitioners will receive tailored feedback, benchmarked against all other participants. By undertaking multiple iterations of evaluation and feedback, those delivering exercise training for older people with sarcopenia or frailty will be able to share learning and evolve practice towards the optimal duration, modality, intensity, frequency and measures to improve outcomes for older people living with sarcopenia or frailty.

\section{KNOWLEDGE AND ATTITUDES TO RECOVERY FROM RESISTANCE EXERCISE IN OLDER ADULTS AT RISK OF SARCOPENIA}

Eleanor Jayne Hayes ${ }^{1}$, Antoneta Granic ${ }^{1,2 \dagger}$, Christopher Hurst ${ }^{1,2 \dagger}$, Lorelle Dismore ${ }^{1,2}$, Avan A. Sayer ${ }^{1,2}$, Emma Stevenson $^{3}$

'AGE Research Group, Translational and Clinical Research Institute, Newcastle University, Newcastle upon Tyne, United Kingdom

${ }^{2}$ NIHR Newcastle Biomedical Research Centre, Newcastle upon Tyne Hospitals NHS Foundation Trust and Newcastle University, Newcastle upon Tyne, United Kingdom

${ }^{3}$ Newcastle University, Population Health Sciences Institute, Newcastle upon Tyne, United Kingdom

'equal contribution

Background: Resistance exercise can improve muscle strength and physical performance in older adults and is recommended as a first-line treatment for sarcopenia. Despite the long-term benefits, resistance exercise can cause unfavourable short-term side effects such as transient fatigue and exercise induced muscle damage. Our aim was to understand the knowledge and attitudes to recovery from resistance exercise in older adults at risk of sarcopenia.

Methods: 291 older adults ( $74 \pm 4$ years) were recruited through social media advertising, and patient and public involvement platforms, to complete a self-administered online survey composed of open-ended and multiplechoice questions about their understanding of exercise induced muscle damage and recovery. Content analysis was conducted on responses to open-ended questions through a systematic classification process of coding to identify themes and patterns.

Results: A general misunderstanding of muscle damage and muscle soreness was identified in our participants. A common misconception was that muscle damage and soreness were due to excessive exercise, injury, or poor technique (e.g., "Over exercising or doing it the wrong way can cause torn muscles and ligaments, and back problems"), rather than being an expected consequence of resistance exercise. Furthermore, 152/291 (52\%) of respondents said that they would be discouraged to at least some extent from completing their usual daily activities if they were experiencing muscle soreness until the pain had subsided while 195/291 (67\%) said muscle soreness would discourage them from completing further exercise.

Conclusions: There is a need to educate older adults on the expected short-term effects of resistance exercise. Reassurance that muscle damage and soreness are not due to incorrect exercise practices, but a normal component of the training process, may help to support ongoing engagement in resistance exercise programmes.

\section{ZOOMing INTO THE VIRTUAL WORLD: REMOTE DATA COLLECTION IN THE MASS_ LIFECOURSE STUDY}

Christopher Hurst' ${ }^{1,2}$, Susan J. Hillman ${ }^{1,2}$, Antoneta Granic ${ }^{1,2}$, Terry J. Aspray ${ }^{1,2}$, Avan A. Sayer ${ }^{1,2}$, Richard M. Dodds ${ }^{1,2}$

'AGE Research Group, Translational and Clinical Research Institute, Faculty of Medical Sciences, Newcastle University, Newcastle, UK ${ }^{2}$ NIHR Newcastle Biomedical Research Centre, Newcastle University and Newcastle upon Tyne NHS Foundation Trust

Background: The Muscle Ageing and Sarcopenia Studies Lifecourse (MASS_Lifecourse) project aims to recruit a cohort of adults aged 45-85 to investigate age-related changes in skeletal muscle. Study participation consists of a home-based health and lifestyle assessment and a clinic visit involving a range of physical measures. In 2020 , restrictions imposed in response to the coronavirus pandemic meant that home-based assessments were impractical or a barrier to participation and alternative data collection approaches were needed. This work aims to consider the feasibility and acceptability of conducting these assessments using a video-conferencing system.

Methods: After responding to a written invitation, prospective participants were contacted to confirm eligibility and asked if they were willing to complete the home-based assessment virtually using Zoom or Microsoft Teams. Assessments were arranged with participants who were emailed a meeting link in advance. Participants were briefed via telephone about how to join the virtual meeting and advised to find a quiet, comfortable space. Prompts for multiple choice questions were shown using Microsoft PowerPoint via screen sharing. Results: All participants ( $n=6$; age $53-75$ years) who were asked to complete a virtual assessment agreed ( $5 \times$ Zoom; $1 \times$ Microsoft Teams). Assessment duration ranged from 39-62 minutes which was similar to previous home-based interviews in the study. Virtual assessments were acceptable to participants who were positive about their experiences. Participants found the technology easy to use and indicated that they felt comfortable engaging in the study from home. Some participants suggested that this approach was more 
convenient for them compared with a traditional home visit. Conclusion: Performing virtual assessments was feasible and acceptable to participants. These findings should encourage researchers to explore the potential of virtual data collection. This approach needs to be explored in a wider range of participants, particularly in those less familiar with relevant technology.

\section{THE NORTH EAST FRAILTY SCORE - A NEW COMPREHENSIVE FRAILTY TOOL FOR ELECTIVE CARDIAC SURGERY PATIENTS - RESULTS OF THE PILOT STUDY}

Lu Wang ${ }^{1,2}$, Greta Wood ${ }^{2}$, Victoria White ${ }^{1}$, Kelly Bertram ${ }^{1}$, Sandra Laidler ${ }^{1}$, Stephen C. Clark ${ }^{1}$, Belgin Ozalp',

Karen L. Booth ${ }^{1}$

'Newcastle upon Tyne Hospitals NHS Foundation Trust, Newcastle upon Tyne, UK

${ }^{2}$ Newcastle University, Newcastle upon Tyne, UK

Background: Frailty has been recognised as a risk factor of mortality and morbidity post-surgery. The currently available frailty scores only assess one or selected few of its components. This pilot study aims to assess the feasibility of comprehensively measuring frailty of elective cardiac surgery patients.

Methods: From December 2018 to September 2020, all cardiac surgery patients $\geq 70$ years underwent a comprehensive frailty assessment pre-operatively. 1 point each was assigned for slow gait speed, upper and lower extremity weakness, weight loss, exhaustion, anaemia, hypoalbuminaemia, malnutrition, cognitive impairment, and reduced activities of daily living and instrumental activities of daily living. Patients with a score $\geq 4$ out of 11 points were deemed as frail. All of the patients were followed up for at least 90 days. Kaplan-Meier survival analysis and logistic regression were used to study whether the frailty score predicts outcomes.

Results: $82.1 \%$ (165/201) consecutively recruited patients completed the full assessment and underwent cardiac surgeries in the study period. 38.2\% (63/165) patients, who were deemed frail, had increased risk of developing major complications (frail vs. non-frail: $23.8 \%$ (15/63) vs. $6.9 \%$ (7/102), p=0.002), longer ITU (2 [1 $4]$ vs. 1 [1-3] days, $p=0.002$ ) and hospital stay ( 8 [6-13] vs. 6 [5-7] days, $p<0.001$ ), and poorer post-operative survival (log-rank test $\mathrm{p}=0.002)$. Moreover, frailty predicts major complications (odds ratio (OR) 3.66, 95\% confidence interval ( $\mathrm{Cl}) 1.27-10.54)$, ITU stay $>3$ days (OR 3.48, 95\% Cl 1.37-8.88) and hospital stay >10 days (OR 2.66, 95\% Cl 1.14-6.22), independent of age, sex, body mass index and EuroSCORE II.

Conclusions: It is feasible to comprehensively evaluate cardiac surgery patients' frailty using the North East frailty score. This frailty score is superior to EuroSCORE
II in predicting post-cardiac surgery outcomes. Although further validation is required, this frailty score has the potential to facilitate the heart team to better risk stratify patients.

\section{DIETITIANS' REPORTED BARRIERS AND ENABLERS TO PRESCRIBING EXERCISE IN OLDER ADULTS \\ Stacey Jones, Deborah Lycett, Bernice Tighe, Michael Duncan \\ Coventry University, Coventry, England}

Background: Combined nutrition and exercise interventions are recommended for the prevention and treatment of sarcopenia. Dietitians are considered experts in nutrition, but not often providers of advice on exercise; however dietitians are in an ideal position to maximise every opportunity to provide comprehensive, holistic advice and ultimately reduce the amount of professionals patients have to engage with. This research explored the barriers and enablers reported by dietitians' to prescribing exercise.

Methods: Following ethical approval, a qualitative methodology employed focus groups, using purposive and convenience recruitment. Focus groups were audio recorded and transcribed verbatim. Data were analysed inductively using Braun and Clarke's thematic analysis. Data were coded using latent and semantic coding, and then grouped into similar themes.

Results: Four themes emerged from the data: i) existing practice, ii) stakeholder influence on role determinants, iii) pre-requisites for an extended role in exercise prescription, iv) the future emerging role. Perceived barriers included limited knowledge and confidence in providing specific exercise interventions, limited knowledge of assessing exercise capacity and contraindications, lack of time including the need to prioritise nutrition advice, perceived risk of causing harm to patient, and concerns around professional indemnity and liability. Perceived enablers included training, guidance and resources, support from managers and professional bodies, closer multidisciplinary team working, and being able to demonstrate effectiveness by measuring outcomes.

Conclusion: Physical activity interventions should be included for all older adults at risk of sarcopenia as an early intervention for prevention, therefore, dietitians should offer advice on physical activity as part of the Making Every Contact Count initiative as a minimum. A specific screening tool for sarcopenia could identify those at risk and allow a targeted approach to prevention. If dietitians are to confidently discuss physical activity with patients, significant barriers in working practice need to be overcome and additional training needs met. 


\section{UNDERSTANDING ETHNICITY DRIVEN DIFFERENCES IN MUSCLE QUANTITY AND QUALITY: DIVERSIFYING REFERENCE POPULATIONS FOR USE OF ULTRASOUND}

Zeinab Majid ${ }^{1,3}$, Carly Welch ${ }^{1,2,3}$, Isabelle Andrews ${ }^{3}$, Zaki Hassan-Smith ${ }^{3,4}$, Vicky Kamwa ${ }^{1,3,4}$, Hannah Picton ${ }^{3}$, Daisy Wilson ${ }^{1,3}$, Thomas A. Jackson ${ }^{1,2,3}$

'Institute of Inflammation and Ageing, University of Birmingham, Birmingham, B $152 \pi$, UK

${ }^{2}$ MRC-Versus Arthritis Centre for Musculoskeletal Ageing Research, University of Birmingham and University of Nottingham, UK

${ }^{3}$ University Hospitals Birmingham NHS Trust, Birmingham, B 15 2GW, UK

${ }^{4}$ Musculoskeletal Endocrinology Research Group, Centre for Endocrinology, Diabetes and Metabolism, Birmingham Health Partners, Birmingham, UK

Background: Ultrasonography is an increasingly recognised pragmatic tool to identify low muscle quantity and assess muscle quality in sarcopenia. Cut off measures for sarcopenia diagnosis differ between European and Asian populations. We aimed to diversify our ultrasound reference population and characterise differences in muscle quantity and quality between ethnic minority groups.

Methods: We carried out an observational study in Black, Asian and Minority Ethnic young healthy volunteers. Ultrasound was used to measure thigh muscle thickness, a measure of Bilateral Anterior Thigh Thickness (BATT). These data were added to an existing, mostly White, reference population of 112 adults used for sarcopenia diagnosis. Ultrasound was performed on all volunteers; the majority had Bioelectrical Impedance Analysis measures taken.

Results: There were 45 volunteers, with a total reference population of 157 . Ninety-seven individuals were White, 18 were Black, 9 were East Asian and 33 were South Asian. Seventy-six were men and 81 were women. The mean (standard deviations) BATT measures were: $6.30 \mathrm{~cm}$ (1.49) white women, $7.46 \mathrm{~cm}(1.16)$ white men; $5.66 \mathrm{~cm}$ (1.07) South Asian women, $7.62 \mathrm{~cm}(1.69)$ South Asian men; $6.57 \mathrm{~cm}$ (1.09) East Asian women, $7.30 \mathrm{~cm}$ (0.95) East Asian men and $7.30 \mathrm{~cm}(1.46)$ black women and $8.51 \mathrm{~cm}$ (1.49) black men. Mean BATT was higher in Black individuals, $8.11 \mathrm{~cm}(1.55)$ compared to South Asians, $6.64 \mathrm{~cm}(1.71)$, with a mean difference of $1.47 \mathrm{~cm}(0.44)$, $\mathrm{p}=0.006$. They also had higher skeletal muscle mass $(+1.03 \mathrm{~kg}, \mathrm{Cl} 0.87$ to $1.98, \mathrm{p}=0.03)$ compared to South Asians. Of the three minority groups, East Asians had the highest echogenicity, and black participants had the lowest, suggesting better quality of muscle.

Conclusion: We have demonstrated differences in muscle quantity and quality between ethnicities. We propose reference populations in Europe used for cut-off points in sarcopenia diagnosis are actively diversified to include ethnicity driven differences observed in young healthy adults, to improve diagnostic validity. 


\section{APPENDIX}

List of authors and abstract numbers.

Name

Abstract number(s)

A

Adamson S

Alonso-Perez J

Ammour $\mathrm{N}$

Andrews I

Arkesteijn M

Arnal-Gomez

Arnera V

Ashe MC

Aspray TJ

Avenell A

Avramoudas S

B

Baird P

Balasch-Bernat M

Bambrick P

Band MM

Barbera JA

Barker $F$

Barreiro E

Becker C

Beckmann M

Bellantuono I

Bertram K

Birkbeck MG

Blamire A

Bloemink M

Booth KL

Brien S

Buckland C

Bunn S

Butler T

Byrne T

C

Carrasco-Rozas A

Caulfield B

Caulfield L

Cebria i Iranzo MA

Celis-Morales C

Cezon-Serrano N

Chan K

Chawner M

Cheung A

Chikusu CMP

Chiu TC

Christaki E

Chudasama $Y$

Clegg A

\section{6,7}

21

4

45

17

27,28

4

15

$9,10,42$

6,7

14

18

27,28

2

6,7

22

3,31

22

4

17

20

43

9

1

19

43

15

40

30

38

2

21

4

40

27,28

24

27,28

12

40

15

37

16

5

35

39
Cooke J

Cooper R

Cox NJ

Clark SC

Cuthbertson DJ

D

Dartee W

Davies IG

Davies MJ

De Biase $\mathrm{S}$

Denaxas S

Diaz-Manera J

Di Leo V

Dionyssiotis $Y$

Dismore L

Dodds RM

Donnan PT

Dotchin C

Dowling $\mathrm{L}$

Draper J

Duncan M

E

Elameer M

English $\mathrm{P}$

Erkkila A

F

Farmaki AE

Fatemifar G

Ferguson LD

Fernandez-Simon E

Finan C

Forcano S

Francis $P$

Funnell L

G

Galanos A

Gallardo E

Gani A

Garcia-Aymerich J

Garfield V

Gartrell R

George J

Giangregorio LM

Gillies C

Goljanek-Whysall K

Gomes T

Gorman GS

Gostage J

Granic A

Gray SR

Grant E

Groumas N

Guehring $\mathrm{H}$

Gvalda M
2

$29,32,34$

8

43

33

4

38

35

40

32

21

11

5

10,41

$9,23,29,34,39,42$

6,7

30

33

17

44

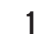

1

25

32

32

24

21

32

27,28

13

15

5

21

1

4

32

18

6,7

15

35

20

11

11

20

$9,10,23,29,31,34,41,42$

24

2

5

4

14 
H

$\begin{array}{lc}\text { Habiballa L } & 23 \\ \text { Hache T } & 4 \\ \text { Hapca A } & 6,7 \\ \text { Harrison M } & 2 \\ \text { Hart A } & 3,31 \\ \text { Harvey S } & 19 \\ \text { Hassan-Smith Z } & 45 \\ \text { Hayes EJ } & 41 \\ \text { Heslop P } & 39,40 \\ \text { Hillman SJ } & 9,29,34,42 \\ \text { Ho FK } & 24 \\ \text { Hope SV } & 39 \\ \text { Hruby A } & 23 \\ \text { Hudson G } & 11 \\ \text { Hume C } & 6,7\end{array}$

Hurst C

I

IIla I

Inderjeeth C

12

Isanejad M

Isaranuwatchai W

25

15

$J$

Jackson M 4

Jackson TA 45

Jain R 15

James J $\quad 37$

Jansen S 12

Johnson MI 13

Jones G 13

Jones $\mathrm{S} \quad 44$

Jordan N 14

Jurk D 23

K

Kamwa V 45

Keller $\mathrm{H} \quad 15$

Kemp P 6,7

Khunti K $\quad 35$

Kirkland J 23

Kirwan R 38

Kisoli A 30

Kiss N 18

Kosmidis $\mathrm{P}$

Kritikaki E 22

Kroger $\mathrm{H} \quad 25$

Kwok R 12

L

Lagnado A 23

Lane $\mathrm{K} \quad 38$

Lai WC $\quad 16$

Laidler S 43

Langford D $\quad 17$

Lawless C 11

Lawson C 35
Lazarou E 5

Lee SC 16

Lewis EG 30

Lin YS 16

Loong D 15

Lu J 12

Lumley HA 1

Lycett D 44

Lyons L 17

M

Majid Z 45

Mazidi M 38

Mazza C 4

McArdle A 25

McCarthy M 4

McCloskey E 20

McDonald C 39

McKenzie E 6,7

Meinke $P \quad 21$

Mickute M

Miller R 4

Milligan J 15

Mitra D 1

Moore SA 1

Mourtzakis M 15

Mueller A 4

Mulcahy R 2

Mupangati YM 26

Murray J 34

N

Narayanan A $\quad 37$

Newman J 11

Norris T 35

0

Ogrodnik M 23

Ozalp B 43

$P$

Padget K 22

Papadatou MC 5

Papaioannou A 15

Papanastasiou C 5

Passos JF 23

Peinado VI 22

Pell JP 24

Perez de Heredia F 38

Petermann-Rocha F 24

Phelan N 2

Pickett S 11

Picton $\mathrm{H} \quad 45$

Piedrafita N 36

Pilvinyte K 6,7

Pinol-Jurado P $\quad 21$

Pinto Pereira SM 32

Price $\mathrm{Cl}$ 
$\mathbf{R}$

Raymond W 12

Razieh C 35

Rikkonen T 25

Roberts HC 8

Robertson F 11

Robinson SM 8,29,34

Rochester L 4

Rodgers $\mathrm{H} \quad 1$

Rodrigues IB $\quad 15$

Rodriguez-Garcia C 38

Rodriguez-Gomez I 24

Romero-Rodriguez MA $\quad 36$

Roubenoff R 4

Rowlands AV 35

Russell OM 11

S

Sargeant J 35

Sattar N 24

Sayer AA $\quad 3,8,9,10,23,29,31,34,39,40,41,42$

Schoser B 21

Sentandreu-Mano T 27,28

Siebert S 24

Simoes DCM 22

Sirola J 25

Smith AC $\quad 35$

Smith F 1

Smith K $\quad 6,7$

Smithard D $\quad 39$

Stastna J 19

Stevenson E $\quad 10,41$

Storey B 39

Straus $S \quad 15$

Strudwick A 19

Struthers AD $\quad 6,7$

Suarez-Calvet X 21

Sumukadas D $\quad 6,7$

T

Tajik B 25

Tan AL 39

Tan IJ 16

Tanamas J 26

Thabane L 15

Thornhill A 39

Thornley I 13

Tighe B 44

Ting J 12

Tomlinson DJ 32

Tortosa-Chulia MA $\quad 27,28$

Tu YH 16

Tumbapo S 19

Tuppen HA 11
V

Vasquez-Oderiz ML $\quad 36$

Vereijken B 4

Viceconti M 4

Vincent AE 11

Von Zglinicki T 23

w

Wagler JB 15

Walker $\mathrm{R} \quad 30$

Walsh J 33

Wang CC 16

Wang L 43

Webb D $\quad 35$

Welch C 45

Weston ZJ 15

Wickramasinghe S 14

Wilkinson TJ 35

Wilson D 45

Wilson T 17

Witham M 3,6,7,39,40

Wood G 43

Wu KY 16

Y

Yarnall A $\quad 4,31$

Yeung $\mathrm{J} \quad 18$

Yates $\mathrm{P}$

Yates T 35

$\mathbf{Z}$

Zaccardi F 35

Zhang $\mathrm{E}$

Zhang X 23 